\title{
Assessing distinct patterns of cognitive aging using tissue- specific brain age prediction based on diffusion tensor imaging and brain morphometry
}

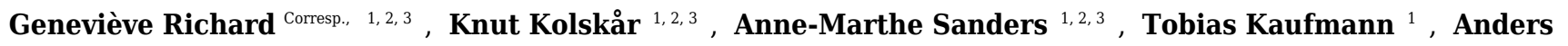
Petersen ${ }^{4}$, Nhat Trung Doan ${ }^{1}$, Jennifer Monereo Sánchez ${ }^{1}$, Dag Alnæes ${ }^{1}$, Kristine M. Ulrichsen ${ }^{1,2,3}$, Erlend S. Dørum ${ }^{1,2,3}$, Ole A. Andreassen ${ }^{1}$, Jan Egil Nordvik ${ }^{3}$, Lars T. Westlye ${ }^{\text {Corresp. } 1,2}$

1 NORMENT, KG Jebsen Centre for Psychosis Research, Division of Mental Health and Addiction, Oslo University Hospital \& Institute of Clinical Medicine, University of Oslo, Oslo, Norway

2 Department of Psychology, University of Oslo, Oslo, Norway

3 Sunnaas Rehabilitation Hospital HT, Nesodden, Norway

${ }^{4}$ Center for Visual Cognition, Department of Psychology, University of Copenhagen, Copenhagen, Denmark

Corresponding Authors: Geneviève Richard, Lars T. Westlye

Email address: genevieve.richard@medisin.uio.no, I.t.westlye@psykologi.uio.no

Multimodal imaging enables sensitive measures of the architecture and integrity of the human brain, but the high-dimensional nature of advanced brain imaging features poses inherent challenges for the analyses and interpretations. Multivariate age prediction reduces the dimensionality to one biologically informative summary measure with potential for assessing deviations from normal lifespan trajectories. A number of studies documented remarkably accurate age prediction, but the differential age trajectories and the cognitive sensitivity of distinct brain tissue classes have yet to be adequately characterized. Exploring differential brain age models driven by tissue-specific classifiers provides a hitherto unexplored opportunity to disentangle independent sources of heterogeneity in brain biology. We trained machine-learning models to estimate brain age using various combinations of FreeSurfer based morphometry and diffusion tensor imaging based indices of white matter microstructure in 612 healthy controls aged 18-87 years. To compare the tissue-specific brain ages and their cognitive sensitivity we applied each of the 11 models in an independent and cognitively well-characterized sample $(n=265,20-88$ years). Correlations between true and estimated age and mean absolute error (MAE) in our test sample were highest for the most comprehensive brain morphometry $(r=0.83$, $\mathrm{Cl}: 0.78-0.86, \mathrm{MAE}=6.76$ years $)$ and white matter microstructure $(r=0.79, \mathrm{Cl}: 0.74-0.83$, $M A E=7.28$ years) models, confirming sensitivity and generalizability. The deviance from the chronological age were sensitive to performance on several cognitive tests for various models, including spatial Stroop and symbol coding, indicating poorer performance in individuals with an over-estimated age. Tissue-specific brain age models provide sensitive measures of brain integrity, with implications for the study of a range of brain disorders. 
Assessing distinct patterns of cognitive aging using tissue-specific brain age prediction based on diffusion tensor imaging and brain morphometry

Geneviève Richarda,b,c,, Knut Kolskår ${ }^{\mathrm{a}, \mathrm{b}, \mathrm{c}}$, Anne-Marthe Sanders ${ }^{\mathrm{a}, \mathrm{b}, \mathrm{c}}$, Tobias Kaufmann ${ }^{\mathrm{a}}$, Anders Petersen $^{\mathrm{d}}$, Nhat Trung Doan ${ }^{\mathrm{a}}$, Jennifer Monereo Sánchez ${ }^{\mathrm{a}}$, Dag Alnæs ${ }^{\mathrm{a}}$, Kristine M.

Ulrichsen ${ }^{\mathrm{a}, \mathrm{b}, \mathrm{c}}$, Erlend S. Døruma, ${ }^{\mathrm{a}, \mathrm{b}, \mathrm{c}}$, Ole A. Andreassen ${ }^{\mathrm{a}}$, Jan Egil Nordvik ${ }^{\mathrm{c}}$, Lars T. Westlye,

${ }^{a}$ NORMENT, KG Jebsen Centre for Psychosis Research, Division of Mental Health and Addiction, Oslo University Hospital \& Institute of Clinical Medicine, University of Oslo, Norway

${ }^{\mathrm{b}}$ Department of Psychology, University of Oslo, Norway

${ }^{c}$ Sunnaas Rehabilitation Hospital HT, Nesodden, Norway

${ }^{\mathrm{d}}$ Center for Visual Cognition, Department of Psychology, University of Copenhagen, Copenhagen, Denmark

\section{*Corresponding authors:}

Geneviève Richard \& Lars T. Westlye

Email: genevieve.richard@medisin.uio.no, 1.t.westlye@psykologi.uio.no

Postal address: Oslo University Hospital, P.O.Box 4956 Nydalen, 0424 OSLO, Norway

Telephone: +47230273 50, Fax: +4723027333 


\section{Abstract}

2 Multimodal imaging enables sensitive measures of the architecture and integrity of the human

3 brain, but the high-dimensional nature of advanced brain imaging features poses inherent

4 challenges for the analyses and interpretations. Multivariate age prediction reduces the

5 dimensionality to one biologically informative summary measure with potential for assessing

6 deviations from normal lifespan trajectories. A number of studies documented remarkably

7 accurate age prediction, but the differential age trajectories and the cognitive sensitivity of

8 distinct brain tissue classes have yet to be adequately characterized. hitherto unexplored opportunity to disentangle independent sources of heterogeneity in brain

11 biology. We trained machine-learning models to estimate brain age using various combinations 12 of FreeSurfer based morphometry and diffusion tensor imaging based indices of white matter 13 microstructure in 612 healthy controls aged $18-87$ years. To compare the tissue-specific brain 14 ages and their cognitive sensitivity we applied each of the 11 models in an independent and 15 cognitively well-characterized sample ( $n=265,20-88$ years). Correlations between true and 16 estimated age and mean absolute error (MAE) in our test sample were highest for the most 17 comprehensive brain morphometry $(r=0.83, \mathrm{CI}: 0.78-0.86, \mathrm{MAE}=6.76$ years $)$ and white matter 18 microstructure $(\mathrm{r}=0.79, \mathrm{CI}: 0.74-0.83, \mathrm{MAE}=7.28$ years $)$ models, confirming sensitivity and 19 generalizability. The deviance from the chronological age were sensitive to performance on 20 several cognitive tests for various models, including spatial Stroop and symbol coding, indicating 21 poorer performance in individuals with an over-estimated age. Tissue-specific brain age models 22 provide sensitive measures of brain integrity, with implications for the study of a range of brain 23 disorders. 


\section{Introduction}

25 Increasing age is a major risk factor for cognitive decline and neurodegeneration, and deviating

26 lifespan trajectories in brain structure and function is a sensitive marker in several common

27 neurological and mental disorders (Cole \& Franke 2017). The maturing and aging brain is highly

28 heterogeneous in term of individual trajectories and in term of brain regions and mechanisms

29 involved (Fjell et al. 2013; Westlye et al. 2010b). Understanding the individual determinants and

30 heterogeneity of the developing and aging brain is imperative for identifying persons at risk for

31 various brain disorders, and for developing and applying effective and targeted treatments.

32 Exploring different modalities acquired by magnetic resonance imaging (MRI) provides a

33 powerful tool to investigate age-related differences in both gray- and white- matter tissue classes

34 across brain regions. However, the richness and complexity of the information provided by

35 advanced imaging pipelines challenges its interpretation. Together, the multifactorial age-related

36 variability and the richness of imaging measures have motivated the development of biologically

37 informative summary measures based on brain imaging data. Using machine learning to estimate

38 the biological age of the brain based on neuroimaging data is one such approach (Cole \& Franke

39 2017; Cole et al. 2018; Kaufmann et al. 2018). Deviation from the normative trajectory is a

40 highly relevant biomarker for the integrity of the brain in healthy and clinical populations

41 (Marquand et al. 2016; Wolfers et al. in press). Brain age gap is a heritable trait showing

42 regionally specific genetic overlaps with major brain disorders, including schizophrenia and

43 multiple sclerosis (Kaufmann et al. 2018), and accumulating evidence supports increased brain

44 age in several clinical groups, including patients with schizophrenia (Kaufmann et al. 2018;

45 Schnack et al. 2016), Alzheimer's disease (Amoroso et al. 2017; Kaufmann et al. 2018), HIV

46 (Cole et al. 2017b; Kuhn et al. 2018), multiple sclerosis (Kaufmann et al. 2018), and 
47 cardiovascular risk factors (Franke et al. 2013; Habes et al. 2016). Indeed, while individuals with

48 brains estimated as younger than their chronological age have been shown to be more physically

49 active (Steffener et al. 2016), augmented brain age has been associated with poor health (Ronan

50 et al. 2016), poor cognitive performance (Liem et al. 2017), early neurodegenerative diseases

51 (Gaser et al. 2013), and increased mortality (Cole et al. 2017a). Less is known about the

52 biological and regional heterogeneity, i.e. to which degree different brain regions, systems or

53 compartments show differential aging patterns and sensitivity to cognitive performance. Brain

54 gray and white matter compartments, which can be assessed and quantified using T1-weighted

55 imaging and diffusion tensor imaging (DTI), respectively, comprise distinct tissue classes with

56 largely differential biological and environmental modifiers and age trajectories (Bennett et al.

57 2010; Cao et al. 2017; Fjell et al. 2013; Salat et al. 2005; Storsve et al. 2014; Westlye et al.

58 2010a; Westlye et al. 2010b). Therefore, allowing for differential brain age models for these

59 distinct classes provides an opportunity to disentangle independent sources of heterogeneity in

60 brain aging.

61 Thus, to identify common and unique aging patterns with potentially differential

62 sensitivity to cognitive function, we aimed to test the complementary value of tissue-specific

63 prediction by comparing brain age estimated using different combinations of FreeSurfer based

64 morphometric measures (regional cortical thickness, surface area and volume) and white matter

65 microstructure (DTI based fractional anisotropy and mean, radial and axial diffusivity) across the

66 brain. Based on previous studies on brain aging, we expected high accuracy and generalizability

67 of the age prediction models (Cole \& Franke 2017). Since tissue specific brain age models

68 capture biologically distinct information, we anticipated that the different FreeSurfer based brain

69 morphometry and white matter microstructure models would only partly reflect common 
70 variance, and therefore provide complementary information with differential sensitivity to

71 cognitive performance. Given that brain age predictions might be sensitive to the overall

72 integrity of the brain (Liem et al. 2017), we anticipated that adult individuals in the targeted age

73 range who show and over-estimated brain age would also show lower cognitive performance, in

74 particular among the elderly, and that the tissue-specific brain age models would show partly

75 differential cognitive sensitivity.

76 To ensure generalizability, we trained the models in a large publicly available training set

$77(\mathrm{n}=612,18-87$ years $)$ and validated their performance using 10-fold cross-validation before

78 applying to an independent and well characterized test set ( $\mathrm{n}=265,20-88$ years). We assessed the

79 cognitive sensitivity using linear and non-linear models with performance on a range of paper-

80 and-pencil and computerized tests comprising different large-scale cognitive domains

81 (processing speed, executive functioning, working memory, attention, and general intellectual

82 abilities) and cognitive scores based on computational models as dependent variables and age,

83 sex and brain age gap (BAG, estimated brain age minus chronological age) as independent

84 variables. For transparency, we report results both at an uncorrected level and corrected using

85 false discovery rate (FDR) and Bonferroni methods to control the error rate.

86

87 Materials and methods

88 Figure 1 displays a flowchart of the main analysis pipeline. Table 1 summarizes key

89 demographics. We included data from healthy volunteers from two independent cohorts: (1) the

90 Cambridge Centre for Ageing and Neuroscience (Cam-CAN) sample (http://www.mrc-

91 cbu.cam.ac.uk/datasets/camcan/; (Shafto et al. 2014; Taylor et al. 2017)) and (2) StrokeMRI,

92 which is an ongoing study on the determinants of stroke recovery, brain health and successful 
93 aging (Dorum et al. 2016; Dorum et al. 2017). Figure 2 shows the age distribution for each

94 sample. The distribution of age $(t=-2.09, p=0.037)$ and $\operatorname{sex}\left(\chi^{2}(1)=10.92, p<0.001\right)$ differed 95 between samples.

96 Volunteers were recruited to Cam-CAN through a large-scale collaborative research 97 project funded by the Biotechnology and Biological Sciences Research Council (BBSRC, grant 98 number BB/H008217/1), the UK Medical Research Council and University of Cambridge. For more information, see www.cam-can.org. Among the 650 datasets made available, 17 were excluded based on missing or poor quality DTI data and 21 due to poor T1-weighted data quality. Data from the remaining 612 individuals (age 18-87, mean $=54.41, \mathrm{SD}=18.26,314$ females) were included. Healthy individuals were recruited to StrokeMRI through advertisement in newspapers, social media and word-of-mouth. All participants completed a comprehensive cognitive assessment, multimodal MRI and blood sampling for clinical biochemical analysis, various biomarkers and genotyping. MRI and cognitive assessments were performed on two subsequent days. Exclusion criteria included history of stroke, dementia, or other neurologic and psychiatric diseases, alcohol- and substance abuse, medications significantly affecting the nervous system and counter indications for MRI. In addition, individuals scoring lower than 25 on the Montreal

110 Cognitive Assessment (MoCA; Nasreddine et al. 2005) were assessed for inclusion based on 111 their age, level of education and performance on other cognitive tests. No participants were 112 excluded based on a single low score. A neuroradiologist reviewed all scans and 14 participants 113 with clinically significant abnormalities were excluded. Additional exclusion criteria included 114 missing or incomplete MRI or cognitive data $(\mathrm{n}=2)$, or poor quality images $(\mathrm{n}=20)$. The 115 remaining 265 participants (age 20-88, mean $=56.95, \mathrm{SD}=14.84,168$ females) were included in 
116 further analyses. The study was supported by the Norwegian ExtraFoundation for Health and

117 Rehabilitation (2015/FO5146), the Research Council of Norway (249795, 248238), the South-

118 Eastern Norway Regional Health Authority (2014097, 2015044, 2015073), Sunnaas

119 Rehabilitation Hospital, and the Department of Psychology, University of Oslo, and approved by

120 the Regional Committee for Medical and Health Research Ethics (South-East Norway, REK

121 2014/694), and conducted in accordance with the Helsinki declaration. All subjects signed an

122 informed consent prior to participating and received a compensation for their participation.

123

124

- Insert Table 1 and Figure 1 and 2 -

125

126 Cognitive assessment in StrokeMRI

127 Cognitive performance was assessed with a set of neuropsychological and computerized tests

128 assumed to be sensitive to cognitive aging, including the MoCA, the vocabulary and matrix

129 subtests of the Wechsler Abbreviated Scale of Intelligence (WASI; Wechsler 1999), the

130 California Verbal Learning Test (CVLT-II; Delis et al. 2000), and the Delis-Kaplan Executive

131 Function System (D-KEFS) color word interference test (Stroop; Delis et al. 2001). We included

132 several computerized tests from the Cognitive Assessment at Bedside for iPAD (CABPad; Willer

133 et al. 2016), including motor speed, verbal fluency (phonological and semantic), working

134 memory, spatial Stroop (executive control of attention), spatial attention span, and symbol digit

135 coding tests. In addition, in order to assess the specificity of cognitive associations using

136 computation modeling, we included three mathematically independent parameters based on the

137 Theory of Visual Attention (TVA; Bundesen 1990; Bundesen \& Habekost 2008), including

138 short-term memory storage $(K)$, processing speed $(C)$, perceptual threshold $\left(t_{0}\right)$. These 
139 parameters based on computational modeling of response patterns have been shown to be

140 sensitive to age, brain structure and function in healthy individuals (Espeseth et al. 2014;

141 Wiegand et al. 2018) and a range of brain disorders (Habekost 2015; Habekost \& Starrfelt 2009).

142 Here, we used a TVA-based modeling of a whole report (Sperling 1960), in which six letters

143 were briefly presented for different exposure durations and the participant's task was to

144 accurately report as many letters as possible. Task error rate was also assessed (i.e. number of

145 incorrect letters out of reported letters).

146

147 MRI acquisition

148 Cam-CAN participants were scanned on a 3T Siemens TIM Trio scanner with a 32-channel

149 head-coil at Medical Research Council (UK) Cognition and Brain Sciences Unit (MRC-CBSU)

150 in Cambridge, UK. DTI data was acquired using a twice-refocused spin echo sequence with the

151 following parameters a repetition time (TR) of $9100 \mathrm{~ms}$, echo time (TE) of $104 \mathrm{~ms}$, field of view

152 (FOV) of $192 \times 192 \mathrm{~mm}$, voxel size: $2 \mathrm{~mm}^{3}$, 66 axial slices using 30 directions with $\mathrm{b}=1000$

$153 \mathrm{~s} / \mathrm{mm}^{2}, 30$ directions with $\mathrm{b}=2000 \mathrm{~s} / \mathrm{mm}^{2}$, and $3 \mathrm{~b}=0$ images (Shafto et al. 2014). Only the

$154 b=[0,1000]$ were used in the current analysis. High-resolution 3D T1-weighted data was acquired

155 using a magnetization prepared rapid gradient echo (MPRAGE) sequence with the following

156 parameters: TR: $2250 \mathrm{~ms}$, TE: $2.99 \mathrm{~ms}$, inversion time (TI): $900 \mathrm{~ms}$, flip angle: 9, FOV of $256 \mathrm{x}$

$157240 \times 192 \mathrm{~mm}$; voxel size $=1 \mathrm{~mm}^{3}$ isotropic, GRAPPA acceleration factor of 2, scan time 4:32

158 minutes (Shafto et al. 2014).

159 StrokeMRI participants were scanned on a 3T GE 750 Discovery MRI scanner with a 32-

160 channel head coil at Oslo University Hospital. Paddings were used to reduce head motion. DTI

161 data were acquired using an echo planar imaging (EPI) sequence with the following parameters: 
162 TR/TE/flip angle: $8150 \mathrm{~ms} / 83.1 \mathrm{~ms} / 90^{\circ}$, FOV: 256 x $256 \mathrm{~mm}$, slice thickness: $2 \mathrm{~mm}$, in-plane

163 resolution: $2 \mathrm{~mm}, 60$ directions $\left(b=1000 \mathrm{~s} / \mathrm{mm}^{2}\right)$ and $5 \mathrm{~b}=0$ volumes, scan time: 8:58 min. In

164 addition, $7 \mathrm{~b}=0$ volumes with reversed phase-encoding direction were acquired. High-resolution

165 T1-weighted data was acquired using a 3D IR-prepared FSPGR (BRAVO) with the following

166 parameters: TR: $8.16 \mathrm{~ms}$, TE: $3.18 \mathrm{~ms}$, flip angle: $12^{\circ}$, voxel size: $1 \times 1 \times 1 \mathrm{~mm}$, FOV: $256 \times 256$

$167 \mathrm{~mm}, 188$ sagittal slices, scan time: 4:43 minutes.

168

169 DTI processing and analysis

170 Diffusion MRI data from both samples were processed locally using the Oxford Center for

171 Functional Magnetic Resonance Imaging of the Brain (FMRIB) Software Library (FSL)

172 (http://www.fmrib.ox.ac.uk/fsl). To correct for geometrical distortions, motion and eddy

173 currents, data were preprocessed using topup (https://fsl.fmrib.ox.ac.uk/fsl/fslwiki/topup) and

174 eddy (https:/fsl.fmrib.ox.ac.uk/fsl/fslwiki/eddy) respectively (Andersson et al. 2003; Smith et al.

175 2004). Topup uses information from the reversed phase-encoded image, resulting in pairs of

176 images (blip-up, blip-down) with distortions going in opposite directions. From these image pairs

177 the susceptibility-induced off-resonance field was estimated and the two images were combined

178 into a single corrected one (Andersson et al. 2003; Smith et al. 2004). This step was performed

179 on StrokeMRI data only. Eddy detects and replaces slices affected by signal loss due to bulk

180 motion during diffusion encoding, which is performed within an integrated framework along

181 with correction for susceptibility induced distortions, eddy currents and motion (Andersson \&

182 Sotiropoulos 2016). Although these processing steps have been shown to strongly increase the

183 temporal signal-to-noise ratio (tSNR) (Doan et al. 2017), we performed additional visual 
184 inspection to identify and remove poor quality data, such as data that failed the processing steps 185 due to low quality.

186

Fractional anisotropy (FA), eigenvector, and eigenvalue maps were calculated using dtifit

187 in FSL. Mean diffusivity (MD) was defined as the mean of all three eigenvalues, radial diffusivity (RD) as the mean of the second and third eigenvalue, and axial diffusivity (AD) as the principal eigenvalue.

191 Statistics (TBSS; Smith et al. 2006), part of FSL (Smith et al. 2004). First, all subjects' FA data 192 were aligned to a common space using the nonlinear registration tool FNIRT (Andersson et al. 193 2007a; Andersson et al. 2007b). Next, the mean FA image was created and thinned to create a 194 mean FA skeleton, which represents the centers of all tracts common to all participants. Each 195 subject's aligned FA data was then projected onto this skeleton and the resulting data fed into voxelwise cross-subject statistics. The same warping and skeletonization was repeated for MD, $\mathrm{AD}$ and $\mathrm{RD}$. We thresholded and binarized the mean FA skeleton at $\mathrm{FA}>0.2$. For each individual, we calculated the mean skeleton FA, MD, AD and RD, as well as mean values within 23 regions of interest (ROIs) based on two probabilistic white matter atlases provided with FSL, i.e. the CBM-DTI-81 white-matter labels atlas and the JHU white-matter tractography atlas (Hua et al. 2008; Mori et al. 2005; Wakana et al. 2007), yielding a total of 96 DTI features per 202 individual.

\section{T1 processing}

205 T1-weighted images from both samples were processed using FreeSurfer 5.3

206 (http://surfer.nmr.mgh.harvard.edu; (Dale et al. 1999)) including brain extraction, intensity 
207 normalization, automated tissue segmentation, generation of white and pial surfaces (Dale et al.

208 1999). All reconstructions were visually assessed and edited by trained research personnel where

209 appropriate. The reconstructions that failed the corrections were excluded from further analysis,

210 such as data with excessive movement artefacts. Cortical parcellation was performed using the

211 Desikan-Killiany atlas (Desikan et al. 2006; Fischl et al. 2004) and subcortical segmentation was

212 performed based on a probabilistic atlas (Fischl et al. 2002). In addition to global features

213 (intracranial volume, total surface area, whole-cortex mean thickness), mean thickness, total

214 surface area, and volume for each cortical ROI, as well as the volume of subcortical structures

215 were computed yielding a set of 251 FreeSurfer based features.

216

217 Age prediction

218 Eleven different models were trained to estimate age based on the feature sets described above

219 (one based on FreeSurfer T1 features, one based on WM DTI features, one including all T1 and

220 DTI features, in addition to eight models based on a smaller subset of features, including models

221 based on FA, MD, AD, RD, sub-cortical volume, volume, area and thickness to further explore

222 the modality specificity of the estimations).

223 Due to systematic differences in brain features between scanners (Madan 2017) as well as

224 non-linear effects of age, we regressed out main effects of scanner using linear models including

225 age, age squared, sex and scanner for each feature, and used the fitted data in further analysis for

226 brain age prediction. In addition, we regressed out the estimated total intracranial volume from

227 the area and volume features. Next, for each model, we created a training data matrix by

228 concatenating all the features for all participants in the training sample (Cam-CAN), which were

229 used as input to estimate age. We used the xgboost framework in R 
230 (http://xgboost.readthedocs.io/en/latest/R-package/xgboostPresentation.html), an efficient and

231 scalable implementation of gradient boosting machine learning techniques, to build the

232 prediction models. The following parameters were used: learning rate (eta) $=0.1$, nround $=5000$,

233 gamma $=1$, max_depth $=6$, subsample $=0.5$. To estimate the performance of our models, we

234 used a 10-fold cross-validation procedure within the training sample and repeated the cross-

235 validation step 1000 times to provide a robust estimate of model predictive accuracy. Next, we

236 tested the performance of our trained models by predicting age in unseen healthy subjects in the

237 test sample (StrokeMRI).

238 For each feature set, we calculated the correlation between the predicted and the

239 chronological age as a measure of the model performance, in addition to the mean absolute error

240 (MAE, in years). For each individual, we calculated the discrepancy between the estimated and

241 the chronological age, i.e. the BAG, for each model. The MAE was calculated from the BAG for

242 each model. Since we were interested in the effect of BAG independently of age, the effect of

243 age was regressed out for each BAG using linear models.

244

245 Statistical analysis

246 Statistical analysis was performed using R (http://www.r-project.org). For cognitive data, we

247 used outlierTest from the car package (Fox \& Weisberg 2011) to identify the most extreme

248 observations based on a linear model, including age and sex. Twenty-five observations were

249 identified as outliers and treated as missing values based on a Bonferroni corrected $\mathrm{p}<0.05$. To

250 visualize the associations between the cognitive tests and to form cognitive domain scores based

251 on the correlation patterns, we performed hierarchical clustering using the default setting of the

252 heatmap.2 package in gplots (Warnes et al. 2016), which uses hclust (Müllner 2013) to form 
253 clusters based on the complete linkage method. Briefly, this is a step-wise clustering process that

254 merges the two nearest clusters until only one single cluster remains, maximizing distance

255 between individuals components between two clusters.

256 For each cognitive measure and summary score based on the clusters formed form the

257 clustering step above, we used linear models to test for the effect of age and sex. Since cognitive

258 performance may show non-linear associations with age, we performed an additional analysis

259 including both age and age ${ }^{2}$ in the models. Then, for each test showing a significant association

260 with age, we tested whether adding BAG to the models lead to an improved model fit. More

261 specifically, we tested for differential associations with cognitive function by comparing the

262 parameter estimates for the different BAG models using Fisher z-transformation. To test the

263 assumption that increased BAG is more relevant for cognitive function among the elderly, we

264 tested for age by BAG interactions on cognitive performance. For transparency, we report both

265 uncorrected p-values and p-values adjusted using FDR (Benjamini \& Hochberg 1995; Wright

266 1992) and Bonferroni correction using a factor of 495 (11 brain gaps and 45 cognitive features).

267

\section{Results}

269 Brain age prediction

270 10-fold cross-validation on the training sample (Cam-CAN) revealed high correlations between

271 chronological and predicted age for the DTI based white matter microstructure $(\mathrm{r}=0.87)$ and

272 FreeSurfer based morphometric $(\mathrm{r}=0.88)$ models. Likewise, the correlations for FA ( $\mathrm{r}=.76)$, MD

$273(\mathrm{r}=.80), \mathrm{AD}(\mathrm{r}=.83), \mathrm{RD}(\mathrm{r}=78)$, sub-volume $(\mathrm{r}=.84)$, volume $(\mathrm{r}=.80)$, area $(\mathrm{r}=.70)$ and thickness

$274(\mathrm{r}=.79)$ based models also confirmed reasonable model performance. 
276 shows a correlation matrix for the 11 BAGs. Figure 3B shows the correlations between the

277 chronological age and the predicted age in the test sample for each model with their confidence

278 intervals, ranging from $(\mathrm{r}=.86, \mathrm{CI}: .82-.89, \mathrm{MAE}=6.14)$ for the combined model to $\mathrm{r}=.58(\mathrm{CI}: .49$ -

$279.65, \mathrm{MAE}=10.24$ ) for the model based on area. Figure $3 \mathrm{C}$ is described below. Figure 3 (D to F)

280 show the estimated age from the three models that performed best among the 11 feature sets, i.e.

281 the combined DTI and T1 feature models $(\mathrm{r}=.86, \mathrm{CI}: .82-.89, \mathrm{MAE}=6.14)$, the $251 \mathrm{FS}$ T1

282 features $(\mathrm{r}=.83, \mathrm{CI}: .78-.86, \mathrm{MAE}=6.76)$, and the $96 \mathrm{WM}$ DTI features $(\mathrm{r}=.79, \mathrm{CI}: .74-.83$, $283 \mathrm{MAE}=7.28)$.

284

285

- Insert Figure 3 -

286

287

Cognitive assessments and associations with BAGs in StrokeMRI

288 Figure 4 shows a correlation matrix across all normalized cognitive scores with the variables

289 sorted according to the hierarchical clustering used in the main analysis. Several variables were

290 highly correlated, and the clustering solution generally suggested seven broad cognitive domains

291 including (Cluster 1) memory and learning (CVLT, attention span, MoCA), (Cluster 2) visual

292 processing speed (TVA processing speed and perceptual threshold), (Cluster 3) verbal skills

293 (phonological and semantic flow), (Cluster 4) attentional control and speed (spatial Stroop),

294 (Cluster 5) executive control and speed (color-word Stroop), (Cluster 6) reasoning and

295 psychomotor speed (matrix, symbol coding and motor speed, short-term memory storage (TVA-

296 parameter $K)$ ), and (Cluster 7) working memory. Table 1 summarizes descriptive statistics and

297 associations with age and sex for each of the 49 cognitive scores, derived features and domain 
298 scores. Linear models revealed 45 significant associations with age after correcting for multiple

299 comparisons, with the strongest effect sizes for the symbol coding test, motor speed, spatial

300 Stroop and spatial attention span. Since non-linear models revealed significant associations with

301 age $^{2}$ only with the color word Stroop 3 (inhibition) and its derived scores (See supplementary

302 Table S1), the main models presented here are linear in order to keep the model to its simplest

303 form.

304

305

- Insert Figure 4 -

306

307 Table 2 shows summary statistics for the associations between cognitive performance and BAG

308 using linear models, including age and sex as covariates. Figure 5 shows a heatmap of the

309 association between cognitive scores and brain age gaps for which the significant associations

310 have been marked with an asterisk. Supplementary Table S1 and Fig. S1 shows the summary

311 statistics and the heatmap of the associations between cognitive performance and BAG using

312 non-linear models. Figure $3 \mathrm{C}$ shows the mean and standard error of the $45 \mathrm{p}$-values $\left(-\log _{10}(p)\right)$

313 for the cognitive scores and composite scores for each row (i.e. BAGs), with a higher mean

314 representing a stronger cumulative association across tests.

315 Figure 6 shows a scatter plot of the 2 strongest associations, which were found between

316 the most comprehensive model (all features combined) and spatial Stroop congruent trials and

317 number of responses, respectively, indicating poorer performance with higher BAG. Fisher z-

318 transformation revealed no statistically significant differences in the cognitive associations

319 between linear models using tissue-specific BAG. No significant interactions were found

320 between BAG and age on cognitive performance. 


\section{Discussion}

325 Brain aging is highly heterogeneous, and expanding our understanding of the biological

326 determinants of human aging is imperative for reducing the burden of age-related cognitive 327 decline and neurodegenerative disorders. An estimate of an individual's deviation from the 328 expected lifespan trajectory in brain structure and function may provide a sensitive measure of

329 individual brain integrity and health, both in presumably healthy individuals and in patients 330 suffering from various brain disorders.

331 The biological heterogeneity of the brain strongly suggests that the concept of a single

332 brain age is too simple, and that tissue-specific brain age models may provide increased

333 sensitivity and specificity in relation to cognitive and mental functions. In line with this view,

334 our main findings demonstrate that different combinations of FreeSurfer based brain

335 morphometry and DTI based white matter microstructural indices can be used to accurately

336 predict the age of individuals, but that the shared variance from the different models suggest that

337 they reflect partly non-overlapping processes of brain aging. Further, the results revealed partly

338 differential sensitivity to cognitive performance; with the strongest cumulative associations

339 across cognitive tests for brain age gaps estimated using RD. Even though our data provide no

340 strong evidence of independent associations with cognitive performance in the current sample of

341 healthy individuals, tissue specific age prediction models might better inform us about the

342 individual determinants and heterogeneity of the aging brain compared to models collapsing

343 several brain compartments by potentially capturing distinct measures of brain aging. 


\section{Brain age prediction}

346 For the age prediction models, our results demonstrated that the 11 different combinations of

347 FreeSurfer based morphometric measures (regional cortical thickness, surface area and volume)

348 and white matter microstructure features (diffusion tensor imaging (DTI) based fractional

349 anisotropy and mean, radial and axial diffusivity) across the brain age models accurately

350 predicted the age of an individual with a mean absolute error between 6.14 and 10.23 years.

351 Brain morphometry and white matter microstructure models had a MAE of 6.76 and 7.28

352 respectively, which correspond with previous publications (Cole et al. 2016; Han et al. 2014;

353 Valizadeh et al. 2017). In general, combining features and modalities increased the performance,

354 and the highest performing model included a combination of both brain morphometry and white

355 matter microstructure (mean absolute error of 6.14 years). Moreover, the correlations between

356 the different brain age gaps suggested a relatively low level of shared variance (mean correlation

$357=0.51, \mathrm{SD}=0.13)$. Together these findings support the notion that tissue specific brain age

358 models capture biologically distinct information. This is in line with the characteristic lifespan

359 patterns of global linear decreases in gray matter volume and the nonlinear trajectories of total

360 white matter volume and DTI based metrics of white matter microstructure (Cox et al. 2016;

361 Fjell et al. 2013; Ge et al. 2002; Liu et al. 2017; Raz et al. 2010; Westlye et al. 2010b),

362 highlighting that the different compartments carry unique biological information and that

363 combining different modalities lead to a better estimation of the age of individuals (Cherubini et

364 al. 2016; Liem et al. 2017; Madan \& Kensinger 2018).

365

366 Cognitive associations 
367 We performed a comprehensive cognitive assessment of the test sample, confirming previous

368 evidence of substantial age-related differences in cognitive performance across a range of tests

369 and domains. Hierarchical clustering of the cognitive features indicated a characteristic pattern of

370 covariance, largely reflecting broad cognitive domains, including memory and learning, visual

371 processing speed, verbal skills, attentional and executive control, reasoning and psychomotor

372 speed, and working memory. Ninety percent of the included cognitive features showed age-

373 differences, with the largest effect sizes observed for speed-based measures, such as symbol

374 coding test, which measures mental and visuo-motor speed (Willer et al. 2016). This is in line

375 with the well-established literature on age-related decline in information processing speed in

376 healthy aging (Bennett et al. 2010; Craik \& Salthouse 2008; Harada et al. 2013). Importantly, not

377 only tasks measuring reaction time, but also various TVA measures based on computational

378 modeling, such as short-term memory storage $(K)$, processing speed $(C)$, and perceptual

379 threshold $\left(t_{0}\right)$ showed strong associations with age, in line with previous studies (Espeseth et al.

380 2014; Habekost 2015; Habekost et al. 2013; McAvinue et al. 2012; Wiegand et al. 2018).

381 Based on the assumption that brain age captures variance related to the integrity of the

382 brain, we anticipated that adults with an over-estimated age would show lower cognitive

383 performance, and that the tissue-specific brain age models would show partly differential

384 sensitivity. To test these hypotheses, we used linear models to explore the associations between

385 cognitive performance and BAG, with age and sex as covariates, and directly compared the

386 parameter estimates from the different brain age models. We found a significant association

387 between performance on several tests and BAG beyond the age associations, indicating lower

388 performance in individuals with higher BAG. Briefly, one significant association was found for

389 WM DTI, five for combined BAG, two for the sub-volume, one for the RD and one for the MD 
390 BAG. The strongest associations were found with the spatial Stroop congruent trials, and number

391 of responses. These findings support that the deviance between the estimated age and the

392 chronological age captures relevant biological information regarding the cognitive performance

393 of an individual. Whereas we found no significantly different associations between brain age

394 models, the association with symbol digit coding test was only seen for WM DTI BAG, while

395 associations with Stroop 3 and 4 were observed only for sub-volume BAG, suggesting some

396 specificity that should be investigated in future studies including larger samples and a broader

397 spectrum of mental health, cognitive and brain phenotypes, both across healthy and clinical

398 samples. We speculate that the contributions of the different modalities in predicting age and the

399 associations with both cognitive performance, but also age-related illnesses vary across the age-

400 span, as it does during maturational age (Brown et al. 2012). Thus, future studies might benefit

401 from investigating modality specific brain-age estimation using specific age range, including

402 children and adolescents.

403

404 Limitations

405 The present findings do not come without limitations. First, although reducing the dimensionality

406 of complex brain imaging data to a biologically informative brain age is a powerful method to

407 assess deviations from normal lifespan trajectories in brain health, findings from this data

408 reduction method are limited in specificity. Here, we attempted to both reduce the complexity of

409 the information while keeping some modality specificity measured by different MRI parameters.

410 Finding a balance between specificity and precision represents an interesting challenge for future

411 studies. Moreover, causality and individual level trajectories cannot be established based on

412 cross-sectional data. Therefore, future longitudinal studies are needed to inform us about the 
413 relevance of the differential trajectories of the tissue-specific brain age prediction with

414 implications for the study of a range of brain disorders. Next, although the age distribution of the

415 test sample is irrelevant for the individual prediction accuracy, the relative overrepresentation of

416 older individuals in the test sample is a limitation when investigating interactions between BAG

417 and age. Thus, although the lack of brain by BAG interactions on cognitive function did not

418 support our hypothesis that increased BAG is more relevant for cognitive function among the

419 elderly, future studies including individuals across a broader age range and range of function are

420 needed to characterize the lifespan dynamics in the associations between brain and behavior.

421 More specifically, including children and adolescents would be necessary to characterize the

422 transition between development and aging, i.e. the point of inflection from which the sign of the

423 effects are assumed to change, an important phase that requires further investigations. Moreover,

424 although we covered a relatively broad spectrum of structural brain features, the link between

425 imaging based indices of brain structure and brain function is elusive, and brain age models

426 including other brain imaging features, including functional measures, might provide a sensitive

427 supplement to the current models. Lastly, whereas the results showed some numerical

428 differences in the cognitive sensitivity of the different combinations of FreeSurfer based

429 morphometry and white matter microstructure models, these differences were not statistically

430 significant, and the hypothesis that tissue specific models provide increased specificity in terms

431 of cognitive associations remains to be further explored in future studies.

432 In conclusion, we have demonstrated that models based on different combinations of

433 brain morphometry and white matter microstructural indices provide partly differential

434 information about the aging brain, emphasizing the relevance of tissue-specific brain age models

435 in the study of brain and mental function in health and disease. 


\section{References}

437

Amoroso N, Diacono D, Fanizzi A, La Rocca M, Monaco A, Lombardi A, Guaragnella

438 C, Bellotti R, Tangaro S, and Alzheimer's Disease Neuroimaging I. 2017. Deep learning reveals

439 Alzheimer's disease onset in MCI subjects: results from an international challenge. $J$ Neurosci

440 Methods. 10.1016/j.jneumeth.2017.12.011

441 Andersson JLR, Jenkinson M, and Smith S. 2007a. TR07BP1: Non-linear optimisation.

442 FMRIB Analysis Group Technical Reports: FMRIB Analysis Group.

443

Andersson JLR, Jenkinson M, and Smith S. 2007b. TR07JA2: Non-linear registration,

444 aka spatial normalization. FMRIB Analysis Group Technical Reports: FMRIB Analysis Group.

445 Andersson JLR, Skare S, and Ashburner J. 2003. How to correct susceptibility distortions

446 in spin-echo echo-planar images: application to diffusion tensor imaging. Neuroimage 20:870-

447 888. 10.1016/S1053-8119(03)00336-7

448 Andersson JLR, and Sotiropoulos SN. 2016. An integrated approach to correction for off-

449 resonance effects and subject movement in diffusion MR imaging. Neuroimage 125:1063-1078.

$450 \quad$ 10.1016/j.neuroimage.2015.10.019

451 Benjamini Y, and Hochberg Y. 1995. Controlling the False Discovery Rate - a Practical

452 and Powerful Approach to Multiple Testing. Journal of the Royal Statistical Society Series B-

453 Methodological 57:289-300.

454 Bennett IJ, Madden DJ, Vaidya CJ, Howard DV, and Howard JH, Jr. 2010. Age-related

455 differences in multiple measures of white matter integrity: A diffusion tensor imaging study of

456 healthy aging. Hum Brain Mapp 31:378-390. 10.1002/hbm.20872

457 Brown TT, Kuperman JM, Chung Y, Erhart M, McCabe C, Hagler DJ, Jr., Venkatraman

458 VK, Akshoomoff N, Amaral DG, Bloss CS, Casey BJ, Chang L, Ernst TM, Frazier JA, Gruen 
459 JR, Kaufmann WE, Kenet T, Kennedy DN, Murray SS, Sowell ER, Jernigan TL, and Dale AM.

460 2012. Neuroanatomical assessment of biological maturity. Curr Biol 22:1693-1698.

461 10.1016/j.cub.2012.07.002

462 Bundesen C. 1990. A theory of visual attention. Psychol Rev 97:523-547.

463 Bundesen C, and Habekost T. 2008. Principles of Visual Attention: Linking Mind and

464 Brain. Oxford University Press. 10.1093/acprof:oso/9780198570707.001.0001

465 Cao B, Mwangi B, Passos IC, Wu MJ, Keser Z, Zunta-Soares GB, Xu DP, Hasan KM, 466 and Soares JC. 2017. Lifespan Gyrification Trajectories of Human Brain in Healthy Individuals 467 and Patients with Major Psychiatric Disorders. Scientific Reports 7. ARTN 511

468 $10.1038 / \mathrm{s} 41598-017-00582-1$ Cherubini A, Caligiuri ME, Peran P, Sabatini U, Cosentino C, and Amato F. 2016. Importance of Multimodal MRI in Characterizing Brain Tissue and Its Potential Application for 471 Individual Age Prediction. Ieee Journal of Biomedical and Health Informatics 20.

472

473

474

475

476

477

478

479

480

481

10.1109/Jbhi.2016.2559938

Cole JH, and Franke K. 2017. Predicting Age Using Neuroimaging: Innovative Brain Ageing Biomarkers. Trends Neurosci. 10.1016/j.tins.2017.10.001

Cole JH, Marioni RE, Harris SE, and Deary IJ. 2018. Brain age and other bodily 'ages': implications for neuropsychiatry. Mol Psychiatry. 10.1038/s41380-018-0098-1

Cole JH, Poudel RPK, Tsagkrasoulis D, Caan MWA, Steves C, Spector TD, and Montana G. 2016. Predicting brain age with deep learning from raw imaging data results in a reliable and heritable biomarker. ARXIV. 2016arXiv161202572C

Cole JH, Ritchie SJ, Bastin ME, Valdes Hernandez MC, Munoz Maniega S, Royle N, Corley J, Pattie A, Harris SE, Zhang Q, Wray NR, Redmond P, Marioni RE, Starr JM, Cox SR, 
482 Wardlaw JM, Sharp DJ, and Deary IJ. 2017a. Brain age predicts mortality. Mol Psychiatry.

$483 \quad 10.1038 / \mathrm{mp} .2017 .62$

484 Cole JH, Underwood J, Caan MWA, De Francesco D, van Zoest RA, Leech R, Wit 485 FWNM, Portegies P, Geurtsen GJ, Schmand BA, van der Loeff MFS, Franceschi C, Sabin CA, 486 Majoie CBLM, Winston A, Reiss P, Sharp DJ, and Collaboration C. 2017b. Increased brain487 predicted aging in treated HIV disease. Neurology 88:1349-1357.

$488 \quad 10.1212 / \mathrm{Wn} 1.0000000000003790$

489 Cox SR, Ritchie SJ, Tucker-Drob EM, Liewald DC, Hagenaars SP, Davies G, Wardlaw 490 JM, Gale CR, Bastin ME, and Deary IJ. 2016. Ageing and brain white matter structure in 3,513 491 UK Biobank participants. Nat Commun 7:13629. 10.1038/ncomms13629

492 Craik FIM, and Salthouse TA. 2008. The handbook of aging and cognition. New York: 493 Psychology Press.

494 Dale AM, Fischl B, and Sereno MI. 1999. Cortical surface-based analysis - I.

495 Segmentation and surface reconstruction. Neuroimage 9:179-194. DOI 10.1006/nimg.1998.0395

496 Delis DC, Kaplan E, and Kramer JH. 2001. Delis-Kaplan Executive Function

497 System:Technical Manual. San Antonio, TX: Harcourt Assessment Company.

498 Delis DC, Kramer JH, Kaplan E, and Ober BA. 2000. California Verbal Learning Test499 Second Edition (CVLT-II). San Antonio, TX: Psychological Corporation.

500 Desikan RS, Segonne F, Fischl B, Quinn BT, Dickerson BC, Blacker D, Buckner RL, 501 Dale AM, Maguire RP, Hyman BT, Albert MS, and Killiany RJ. 2006. An automated labeling 502 system for subdividing the human cerebral cortex on MRI scans into gyral based regions of 503 interest. Neuroimage 31:968-980. 10.1016/j.neuroimage.2006.01.021 
504 Doan NT, Engvig A, Persson K, Alnaes D, Kaufmann T, Rokicki J, Cordova-Palomera

505 A, Moberget T, Braekhus A, Barca ML, Engedal K, Andreassen OA, Selbaek G, and Westlye

506 LT. 2017. Dissociable diffusion MRI patterns of white matter microstructure and connectivity in

507 Alzheimer's disease spectrum. Scientific Reports 7. ARTN 45131

508

$10.1038 /$ srep45131

509

Dorum ES, Alnaes D, Kaufmann T, Richard G, Lund MJ, Tonnesen S, Sneve MH,

510

Mathiesen NC, Rustan OG, Gjertsen O, Vatn S, Fure B, Andreassen OA, Nordvik JE, and

511 Westlye LT. 2016. Age-related differences in brain network activation and co-activation during

512 multiple object tracking. Brain and Behavior 6. 10.1002/brb3.533

513 Dorum ES, Kaufmann T, Alnaes D, Andreassen OA, Richard G, Kolskar KK, Nordvik

514 JE, and Westlye LT. 2017. Increased sensitivity to age-related differences in brain functional

515 connectivity during continuous multiple object tracking compared to resting-state. Neuroimage

516 148:364-372. 10.1016/j.neuroimage.2017.01.048

517 Espeseth T, Vangkilde SA, Petersen A, Dyrholm M, and Westlye LT. 2014. TVA-based

518 assessment of attentional capacities-associations with age and indices of brain white matter

519 microstructure. Front Psychol 5:1177. 10.3389/fpsyg.2014.01177

520 Fischl B, Salat DH, Busa E, Albert M, Dieterich M, Haselgrove C, van der Kouwe A,

521 Killiany R, Kennedy D, Klaveness S, Montillo A, Makris N, Rosen B, and Dale AM. 2002.

522 Whole brain segmentation: Automated labeling of neuroanatomical structures in the human

523 brain. Neuron 33:341-355. Doi 10.1016/S0896-6273(02)00569-X

524 Fischl B, van der Kouwe A, Destrieux C, Halgren E, Segonne F, Salat DH, Busa E,

525 Seidman LJ, Goldstein J, Kennedy D, Caviness V, Makris N, Rosen B, and Dale AM. 2004. 
526 Automatically parcellating the human cerebral cortex. Cerebral Cortex 14:11-22.

527 10.1093/cercor/bhg087

528 Fjell AM, Westlye LT, Grydeland H, Amlien I, Espeseth T, Reinvang I, Raz N, Holland

529 D, Dale AM, Walhovd KB, and Alzheimer Disease Neuroimaging I. 2013. Critical ages in the

530 life course of the adult brain: nonlinear subcortical aging. Neurobiol Aging 34:2239-2247.

531 10.1016/j.neurobiolaging.2013.04.006

532 Fox J, and Weisberg S. 2011. An $\{\mathrm{R}\}$ Companion to Applied Regression. In: Second, 533 editor. Thousand Oaks, CA: Sage.

534 Franke K, Gaser C, Manor B, and Novak V. 2013. Advanced BrainAGE in older adults 535 with type 2 diabetes mellitus. Frontiers in Aging Neuroscience 5. ARTN 90

536 10.3389/fnagi.2013.00090

537 Gaser C, Franke K, Kloppel S, Koutsouleris N, Sauer H, and Initi AsDN. 2013.

538 BrainAGE in Mild Cognitive Impaired Patients: Predicting the Conversion to Alzheimer's

539 Disease. PLoS One 8. ARTN e67346

540 10.1371/journal.pone.0067346

541

Ge Y, Grossman RI, Babb JS, Rabin ML, Mannon LJ, and Kolson DL. 2002. Age-related

542 total gray matter and white matter changes in normal adult brain. Part I: volumetric MR imaging 543 analysis. AJNR Am J Neuroradiol 23:1327-1333.

544 Habekost T. 2015. Clinical TVA-based studies: a general review. Front Psychol 6:290.

$545 \quad 10.3389 /$ fpsyg.2015.00290

546 Habekost T, and Starrfelt R. 2009. Visual attention capacity: a review of TVA-based

547 patient studies. Scand J Psychol 50:23-32. 10.1111/j.1467-9450.2008.00681.x 
549 Waldemar G. 2013. Visual processing speed in old age. Scand J Psychol 54:89-94.

550 10.1111/sjop.12008

551 Habes M, Guray E, Toledo JB, Zhang T, Bryan RN, Janowitz D, Doshi J, von Sarnowski

552 B, Hegenscheid K, Voelzke H, Schminke U, Hoffmann W, Grabe HJ, and Davatzikos C. 2016.

553 Non-Resilient Brain Aging in Association with Cardiovascular Risk and White Matter

554 Hyperintensities: The Ship Study. Alzheimer's \& Dementia 12:P226-P227.

$555 \quad 10.1016 /$ j.jalz.2016.06.407

556 Han CE, Peraza LR, Taylor JP, and Kaiser M. 2014. Predicting Age across Human

557 Lifespan Based on Structural Connectivity from Diffusion Tensor Imaging. 2014 Ieee

558 Biomedical Circuits and Systems Conference (Biocas):137-140.

559 Harada CN, Natelson Love MC, and Triebel KL. 2013. Normal cognitive aging. Clin

560 Geriatr Med 29:737-752. 10.1016/j.cger.2013.07.002

561 Hua K, Zhang JY, Wakana S, Jiang HY, Li X, Reich DS, Calabresi PA, Pekar JJ, van Zijl

562 PCM, and Mori S. 2008. Tract probability maps in stereotaxic spaces: Analyses of white matter

563 anatomy and tract-specific quantification. Neuroimage 39:336-347.

$564 \quad 10.1016 /$ j.neuroimage.2007.07.053

565 Kaufmann T, van der Meer D, Doan NT, Schwarz E, Lund MJ, Agartz I, Alnæs D, Barch

566 DM, Baur-Streubel R, Bertolino A, Bettella F, Beyer MK, Bøen E, Borgwardt S, Brandt CL,

567 Buitelaar J, Celius EG, Cervenka S, Conzelmann A, Córdova-Palomera A, Dale AM, de

568 Quervain DJF, Di Carlo P, Djurovic S, Dørum ES, Eisenacher S, Elvsashagen T, Espeseth T,

569 Fatouros-Bergman H, Flyckt L, Franke B, Frei O, Haatveit B, Haberg AK, Harbo HF, Hartman

570 CA, Heslenfeld D, Hoekstra PJ, Høgestøl EA, Jernigan T, Jonassen R, Jönsson EG, Kirsch P, 
571 Kloszewska I, Kolskar K-K, Landrø NI, Le Hellard S, Lesch K-P, Lovestone S, Lundervold A,

572 Lundervold AJ, Maglanoc LA, Malt UF, Mecocci P, Melle I, Meyer-Lindenberg A, Moberget T,

573 Norbom LB, Nordvik JE, Nyberg L, Oosterlaan J, Papalino M, Papassotiropoulos A, Pauli P,

574 Pergola G, Persson K, Richard G, Rokicki J, Sanders A-M, Selbæk G, Shadrin AA, Smeland

575 OB, Soininen H, Sowa P, Steen VM, Tsolaki M, Ulrichsen KM, Vellas B, Wang L, Westman E,

576 Ziegler GC, Zink M, Andreassen OA, and Westlye LT. 2018. Genetics of brain age suggest an

577 overlap with common brain disorders. bioRxiv.

578 Kuhn T, Kaufmann T, Doan NT, Westlye LT, Jones J, Nunez RA, Bookheimer SY,

579 Singer EJ, Hinkin CH, and Thames AD. 2018. An augmented aging process in brain white

580 matter in HIV. Hum Brain Mapp. 10.1002/hbm.24019

581 Liem F, Varoquaux G, Kynast J, Beyer F, Masouleh SK, Huntenburg JM, Lampe L,

582 Rahim M, Abraham A, Craddock RC, Riedel-Heller S, Luck T, Loeffler M, Schroeter ML, Witte

583 AV, Villringer A, and Margulies DS. 2017. Predicting brain-age from multimodal imaging data

584 captures cognitive impairment. Neuroimage 148:179-188. 10.1016/j.neuroimage.2016.11.005

585 Liu K, Yao S, Chen K, Zhang J, Yao L, Li K, Jin Z, and Guo X. 2017. Structural Brain

586 Network Changes across the Adult Lifespan. Front Aging Neurosci 9:275.

587 10.3389/fnagi.2017.00275

588 Madan CR. 2017. Advances in Studying Brain Morphology: The Benefits of Open-

589 Access Data. Frontiers in Human Neuroscience 11. ARTN 405

$590 \quad 10.3389 /$ fnhum. 2017.00405

591 Madan CR, and Kensinger EA. 2018. Predicting age from cortical structure across the

592 lifespan. Eur J Neurosci 47:399-416. 10.1111/ejn.13835 
594 Heterogeneity in Clinical Cohorts Using Normative Models: Beyond Case-Control Studies. Biol 595 Psychiatry 80:552-561. 10.1016/j.biopsych.2015.12.023

596 McAvinue LP, Habekost T, Johnson KA, Kyllingsbaek S, Vangkilde S, Bundesen C, and 597 Robertson IH. 2012. Sustained attention, attentional selectivity, and attentional capacity across 598 the lifespan. Atten Percept Psychophys 74:1570-1582. 10.3758/s13414-012-0352-6

Mori S, Wakana S, van Zijl PCM, and Nagae-Poetscher LM. 2005. MRI Atlas of Human 600 White Matter. In: Science E, editor. 1st Edition ed: Elsevier Science. p 276.

601 Müllner D. 2013. \{fastcluster\}: Fast Hierarchical, Agglomerative Clustering Routines for 602 603 $\{\mathrm{R}\}$ and $\{$ Python $\}$. Journal of Statistical Software 53:1-18.

Nasreddine ZS, Phillips NA, Bedirian V, Charbonneau S, Whitehead V, Collin I,

604 Cummings JL, and Chertkow H. 2005. The montreal cognitive assessment, MoCA: A brief 605 screening tool for mild cognitive impairment. Journal of the American Geriatrics Society 606 53:695-699. DOI 10.1111/j.1532-5415.2005.53221.x

607 Raz N, Ghisletta P, Rodrigue KM, Kennedy KM, and Lindenberger U. 2010. Trajectories 608 of brain aging in middle-aged and older adults: regional and individual differences. Neuroimage 609 51:501-511. 10.1016/j.neuroimage.2010.03.020

610 Ronan L, Alexander-Bloch AF, Wagstyl K, Farooqi S, Brayne C, Tyler LK, Fletcher PC, 611 and Cam-CAN. 2016. Obesity associated with increased brain age from midlife. Neurobiology of 612 Aging 47:63-70. 10.1016/j.neurobiolaging.2016.07.010

613 Salat DH, Tuch DS, Greve DN, van der Kouwe AJW, Hevelone ND, Zaleta AK, Rosen 614 BR, Fischl B, Corkin S, Rosas HD, and Dale AM. 2005. Age-related alterations in white matter 
615 microstructure measured by diffusion tensor imaging. Neurobiology of Aging 26:1215-1227.

616 10.1016/j.neurobiolaging.2004.09.017

617 Schnack HG, van Haren NE, Nieuwenhuis M, Hulshoff Pol HE, Cahn W, and Kahn RS.

618 2016. Accelerated Brain Aging in Schizophrenia: A Longitudinal Pattern Recognition Study. Am

619 J Psychiatry 173:607-616. 10.1176/appi.ajp.2015.15070922

620 Shafto MA, Tyler LK, Dixon M, Taylor JR, Rowe JB, Cusack R, Calder AJ, Marslen-

621 Wilson WD, Duncan J, Dalgleish T, Henson RN, Brayne C, Matthews FE, and Cam-CAN. 2014.

622 The Cambridge Centre for Ageing and Neuroscience (Cam-CAN) study protocol: a cross-

623 sectional, lifespan, multidisciplinary examination of healthy cognitive ageing. Bmc Neurology

624 14. ARTN 204

$625 \quad 10.1186 / \mathrm{s} 12883-014-0204-1$

626 Smith SM, Jenkinson M, Johansen-Berg H, Rueckert D, Nichols TE, Mackay CE,

627 Watkins KE, Ciccarelli O, Cader MZ, Matthews PM, and Behrens TEJ. 2006. Tract-based spatial 628 statistics: Voxelwise analysis of multi-subject diffusion data. Neuroimage 31:1487-1505.

629 10.1016/j.neuroimage.2006.02.024

630 Smith SM, Jenkinson M, Woolrich MW, Beckmann CF, Behrens TEJ, Johansen-Berg H,

631 Bannister PR, De Luca M, Drobnjak I, Flitney DE, Niazy RK, Saunders J, Vickers J, Zhang YY, 632 De Stefano N, Brady JM, and Matthews PM. 2004. Advances in functional and structural MR 633 image analysis and implementation as FSL. Neuroimage 23:S208-S219.

$634 \quad 10.1016 /$ j.neuroimage.2004.07.051

635 Sperling G. 1960. The information available in brief visual presentations. Psychological 636 Monographs: General and Applied 74:1-29. 10.1037/h0093759 
638 between chronological and brain age are related to education and self-reported physical activity.

639 Neurobiology of Aging 40:138-144. 10.1016/j.neurobiolaging.2016.01.014

640 Storsve AB, Fjell AM, Tamnes CK, Westlye LT, Overbye K, Aasland HW, and Walhovd

641 KB. 2014. Differential Longitudinal Changes in Cortical Thickness, Surface Area and Volume

642 across the Adult Life Span: Regions of Accelerating and Decelerating Change. Journal of

643 Neuroscience 34:8488-8498. 10.1523/Jneurosci.0391-14.2014

644 Taylor JR, Williams N, Cusack R, Auer T, Shafto MA, Dixon M, Tyler LK, Cam C, and 645 Henson RN. 2017. The Cambridge Centre for Ageing and Neuroscience (Cam-CAN) data 646 repository: Structural and functional MRI, MEG, and cognitive data from a cross-sectional adult 647 lifespan sample. Neuroimage 144:262-269. 10.1016/j.neuroimage.2015.09.018

649 brain anatomical measures. Hum Brain Mapp 38:997-1008. 10.1002/hbm.23434

650

Wakana S, Caprihan A, Panzenboeck MM, Fallon JH, Perry M, Gollub RL, Hua KG, 
Westlye LT, Walhovd KB, Dale AM, Bjornerud A, Due-Tonnessen P, Engvig A,

660 Grydeland H, Tamnes CK, Ostby Y, and Fjell AM. 2010a. Differentiating maturational and

661 aging-related changes of the cerebral cortex by use of thickness and signal intensity. Neuroimage

662 52:172-185. 10.1016/j.neuroimage.2010.03.056

663 Westlye LT, Walhovd KB, Dale AM, Bjornerud A, Due-Tonnessen P, Engvig A,

664 Grydeland H, Tamnes CK, Ostby Y, and Fjell AM. 2010b. Life-Span Changes of the Human

665 Brain White Matter: Diffusion Tensor Imaging (DTI) and Volumetry. Cerebral Cortex 20:2055-

$6662068.10 .1093 /$ cercor/bhp280

667 Wiegand I, Lauritzen MJ, Osler M, Mortensen EL, Rostrup E, Rask L, Richard N,

668 Horwitz A, Benedek K, Vangkilde S, and Petersen A. 2018. EEG correlates of visual short-term

669 memory in older age vary with adult lifespan cognitive development. Neurobiol Aging 62:210-

$670 \quad 220.10 .1016 /$ j.neurobiolaging.2017.10.018

671 Willer L, Pedersen PM, Forchhammer HB, and Christensen HK. 2016. Cognitive

672 assessment at bedside for iPad: A preliminary validation of a novel cognitive test for stroke

673 patients. European Stroke Journal 1:294-301. 10.1177/2396987316665233

674 Wolfers T, Doan NT, Kaufmann T, Alnæs D, Moberget T, Agartz I, Buitelaar J, Ueland

675 T, Melle I, Beckmann CF, Franke B, Andreassen OA, Westlye LT, and Marquand A. in press.

676 Extensive interindividual differences in schizophrenia and bipolar disorder: mapping biological

677 heterogeneity in reference to normative brain ageing JAMA Psychiatry.

678 Wright SP. 1992. Adjusted P-Values for Simultaneous Inference. Biometrics 48:1005-

679 1013. Doi 10.2307/2532694

680

681 


\section{Figure legends}

683 Fig. 1 Flowchart of the main analysis pipeline.

684 Fig. 2 Histogram of the age distribution for each sample.

685 Fig. 3 Comparison between the 11 BAG models. (A) Heatmap of the correlation between

686 different BAGs. (B) Correlations between the chronological age and the predicted age in the test 687 sample for each model with their confidence intervals. (C) Mean and standard error of the 45 p688 values $\left(-\log _{10}(p)\right)$ for the cognitive scores and composite scores for each row (i.e. BAGs), with a 689 higher mean representing a stronger global association across tests. (D) Correlation between the 690 chronological age of each subjects and the combined age, (E) the brain morphometry age, and 691 (F) the white matter microstructure age.

692 Fig. 4 Hierarchical clustering of the cognitive features. Each cognitive score was normalized and 693 when required the scores were multiplied by -1 to ensure that positive scores represent good 694 performance. The higher panel shows the dendrogram resulting from the hierarchical clustering 695 of the scores in 7 cognitive domains. Suppl. table S2 provides detailed overview of all 696 abbreviations used.

697 Fig. 5 Heatmap of the association between cognitive scores and brain age gaps. The color scale 698 depicts the minus $\log$ of the p-values $\left(-\log _{10}(p)\right)$ for each association. The association marked 699 with a small star represents significant associations after FDR correction, and the one marked 700 with a big star shows significant associations after Bonferroni correction. Suppl. table S2 701 provides detailed overview of all abbreviations used.

702

703 Fig. 6 Scatter plots of the 2 strongest associations between cognitive measures and BAG. (A) 704 Association between Spatial stroop congruent reaction time and BAG. (B) Association between 
705 Spatial stroop number of responses and BAG. The color gradient represents the age where lighter

706 color is assigned to older individuals, and darker color to younger individuals. All associations

707 indicate worse performance with higher brain age gap.

708

709

710

711

712

713

714

715

716

717

718

719

720

721

722

723

724

725

726

727 


\section{Table legends}

729 Table 1. Demographics and cognitive information. * significant associations between cognitive

730 measures with age after FDR correction, ** significant associations between cognitive measures

731 with age after Bonferroni correction. IQR: interquartile range. MoCA: Montreal Cognitive

732 Assessment. WASI: Wechsler Abbreviated Scale of Intelligence. CVLT: California Verbal

733 Learning Test. STROOP: Delis-Kaplan Executive Function System (D-KEFS) color word

734 interference test. CP: Cognitive Assessment at Bedside for iPAD (CabPAD). WM: working

735 memory. TVA: Theory of Visual Attention. 1s: longest serie. ss: sum scores. tot: total.

736

737 Table 2. Cognitive associations with Brain Age Gap (BAG) - statistics. * FDR significant **

738 Bonferroni significant. MoCA: Montreal Cognitive Assessment. WASI: Wechsler Abbreviated

739 Scale of Intelligence. CVLT: California Verbal Learning Test. STROOP: Delis-Kaplan

740 Executive Function System (D-KEFS) color word interference test. CP: Cognitive Assessment at

741 Bedside for iPAD (CabPAD). WM: working memory. TVA: Theory of Visual Attention. 1s:

742 longest serie. ss: sum scores. tot: total.

743

744

745

746

747

748

749

750 


\section{Supplementary Materials}

752 Fig. S1. Heatmap of the association between cognitive scores and brain age gaps using non-

753 linear models, including age, age ${ }^{2}$ and sex as covariates. The color scale depicts the minus log of 754 the p-values $\left(-\log _{10}(p)\right)$ for each association. The association marked with a small star represents 755 significant associations after FDR correction, and the one marked with a big star shows 756 significant associations after Bonferroni correction. Suppl. table S2 provides detailed overview 757 of all abbreviations used.

758

759 Table S1. Cognitive associations with Brain Age Gap (BAG) using non-linear models, including 760 age, age ${ }^{2}$ and sex as covariates - statistics. * FDR significant ** Bonferroni significant. MoCA:

761 Montreal Cognitive Assessment. WASI: Wechsler Abbreviated Scale of Intelligence. CVLT:

762 California Verbal Learning Test. STROOP: Delis-Kaplan Executive Function System (D-KEFS)

763 color word interference test. CP: Cognitive Assessment at Bedside for iPAD (CabPAD). WM:

764 working memory. TVA: Theory of Visual Attention. 1s: longest serie. ss: sum scores. tot: total. 765

766 Table S2. List of abbreviations used in Figures 4, 5 and S1. WASI= Wechsler Abbreviated Scale 767 of Intelligence, CLVT= California Verbal Learning Test, STROOP= Delis-Kaplan Executive 768 Function System (D-KEFS) color word interference test, $\mathrm{CP}=$ Cognitive Assessment at Bedside 769 for iPAD (CabPAD), TVA= Theory of Visual Attention. 


\section{Figure 1}

\section{Flowchart of the main analysis pipeline.}

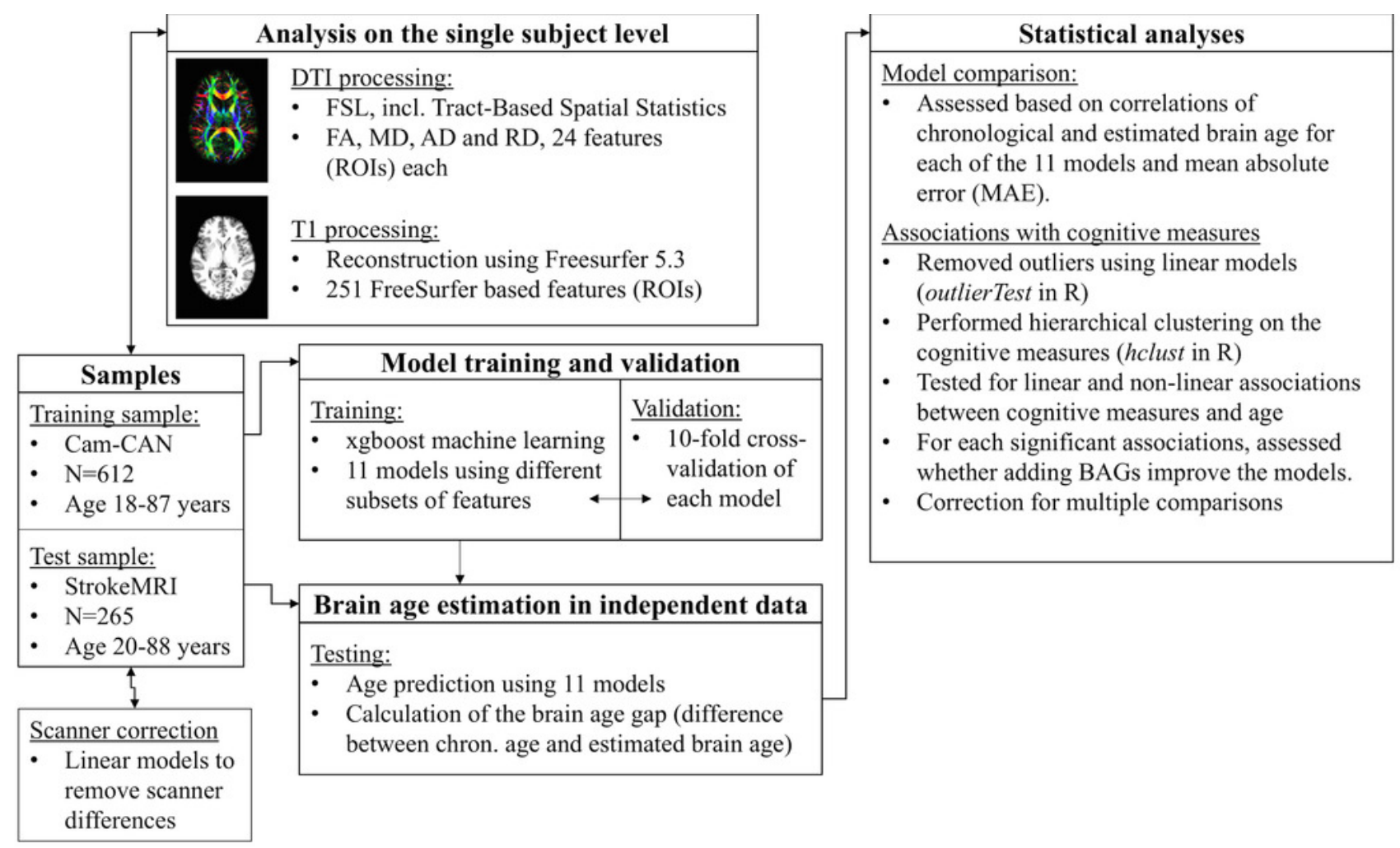


Figure 2

Histogram of the age distribution for each sample.

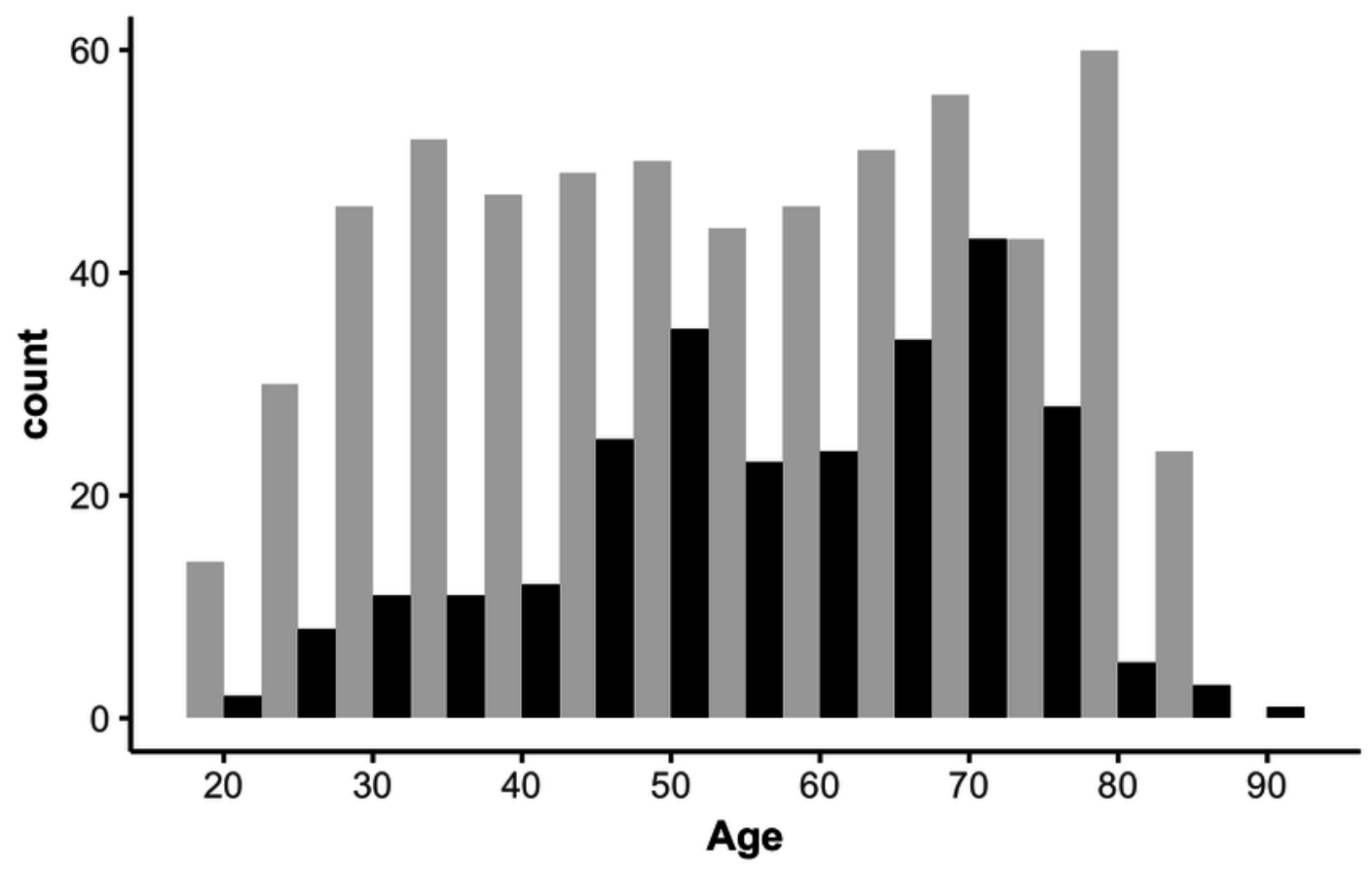

Sample

CAMCAN

STROKEMRI 
Figure 3

Comparison between the 11 BAG models.

(A) Heatmap of the correlation between different BAGs. (B) Correlations between the chronological age and the predicted age in the test sample for each model with their confidence intervals. (C) Mean and standard error of the $45 p$-values $\left(-\log _{10}(p)\right)$ for the cognitive scores and composite scores for each row (i.e. BAGs), with a higher mean representing a stronger global association across tests. (D) Correlation between the chronological age of each subjects and the combined age, (E) the brain morphometry age, and $(F)$ the white matter microstructure age.
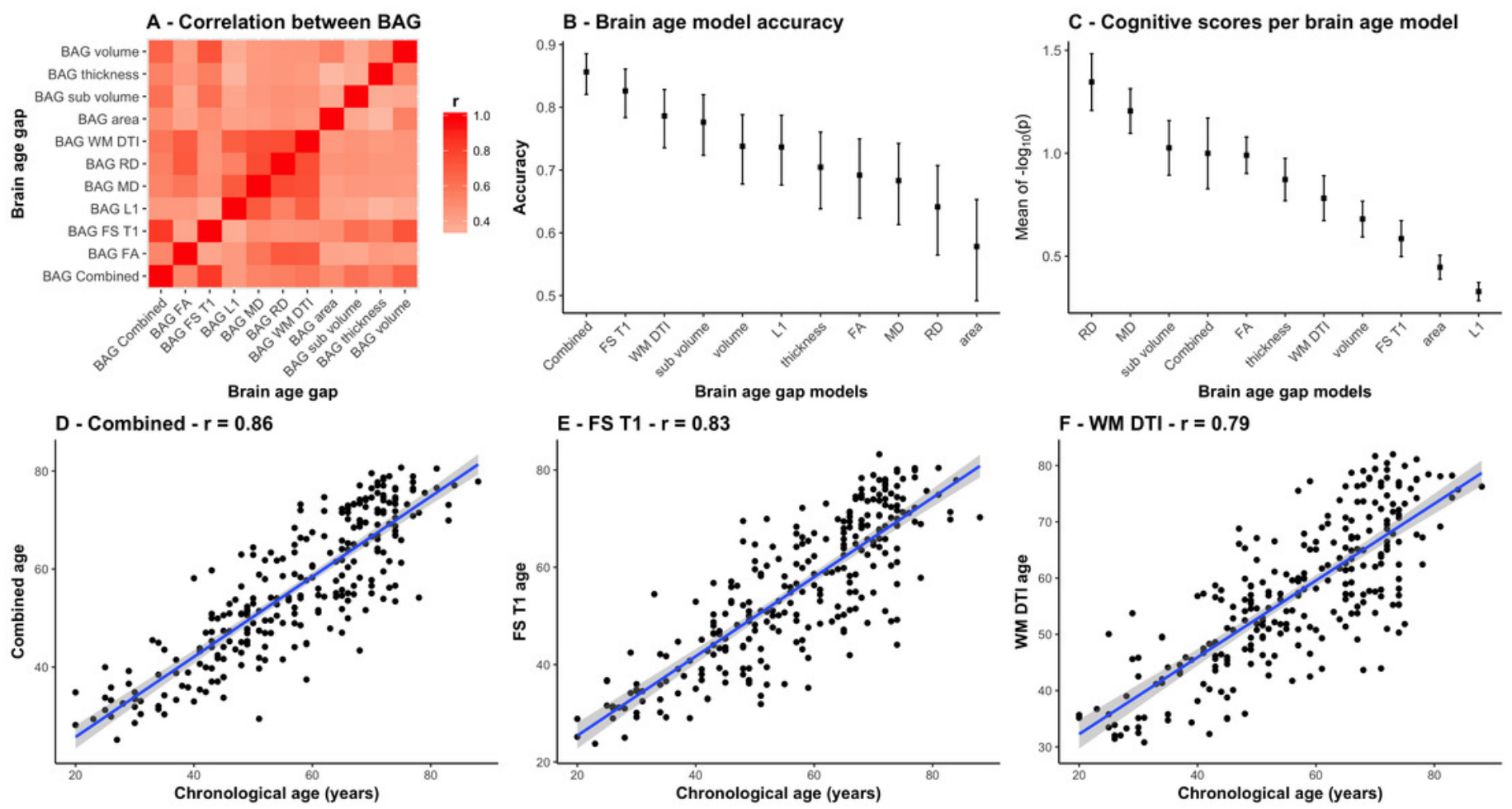
Figure 4 (on next page)

Hierarchical clustering of the cognitive features.

Each cognitive score was normalized and when required the scores were multiplied by -1 to ensure that positive scores represent good performance. The higher panel shows the dendrogram resulting from the hierarchical clustering of the scores in 7 cognitive domains. Suppl. table S2 provides detailed overview of all abbreviations used. 


\section{Figure 5}

Heatmap of the association between cognitive scores and brain age gaps.

The color scale depicts the minus log of the $p$-values $\left(-\log _{10}(p)\right)$ for each association. The association marked with a small star represents significant associations after FDR correction, and the one marked with a big star shows significant associations after Bonferroni correction. Suppl. table $\mathbf{S} 2$ provides detailed overview of all abbreviations used.

\section{Associations between cognitive tests and BAG}

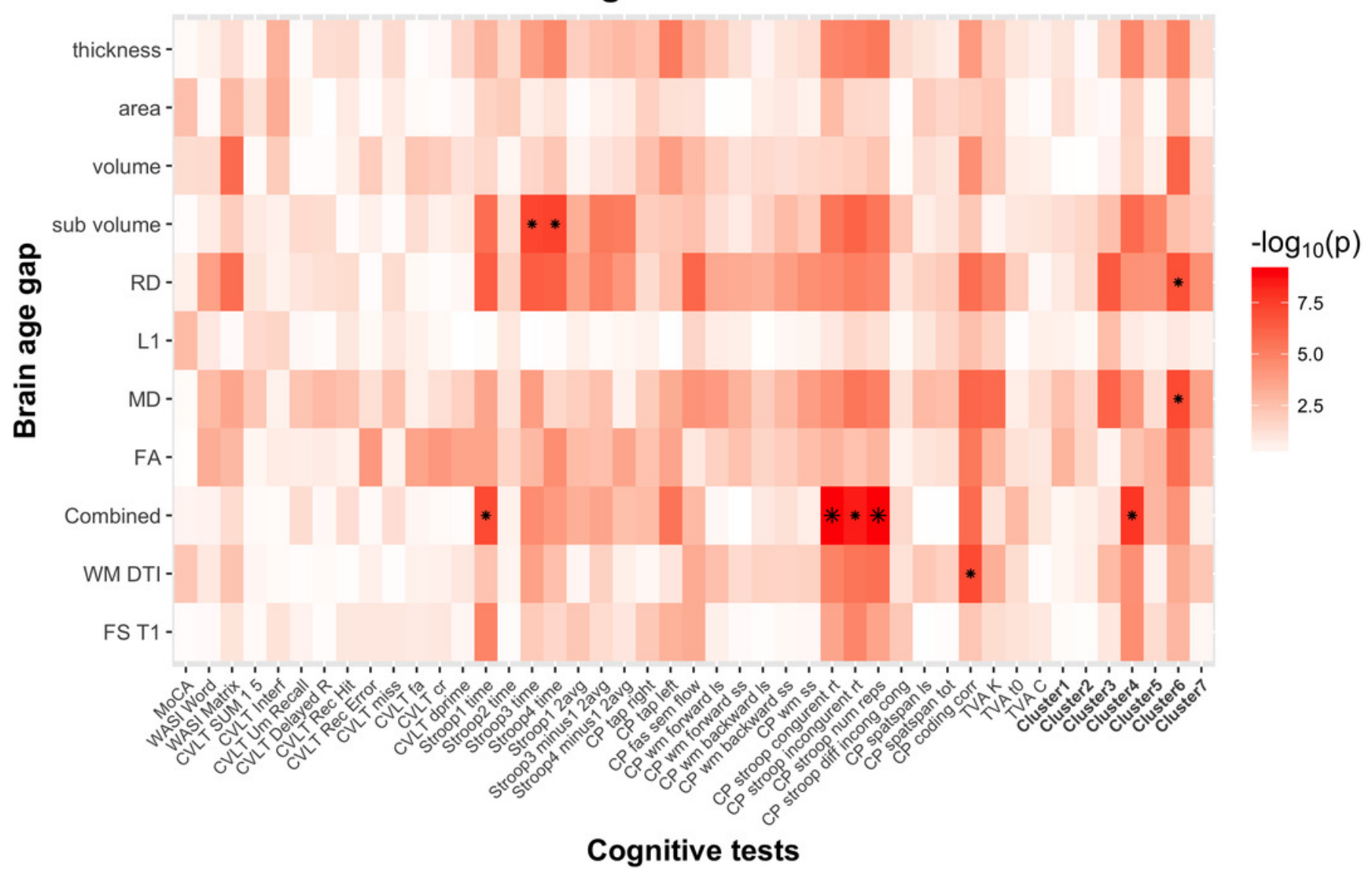


Figure 6

Scatter plots of the 2 strongest associations between cognitive measures and BAG.

(A) Association between Spatial stroop congruent reaction time and BAG. (B) Association between Spatial stroop number of responses and BAG. The color gradient represents the age where lighter color is assigned to older individuals, and darker color to younger individuals. All associations indicate worse performance with higher brain age gap.
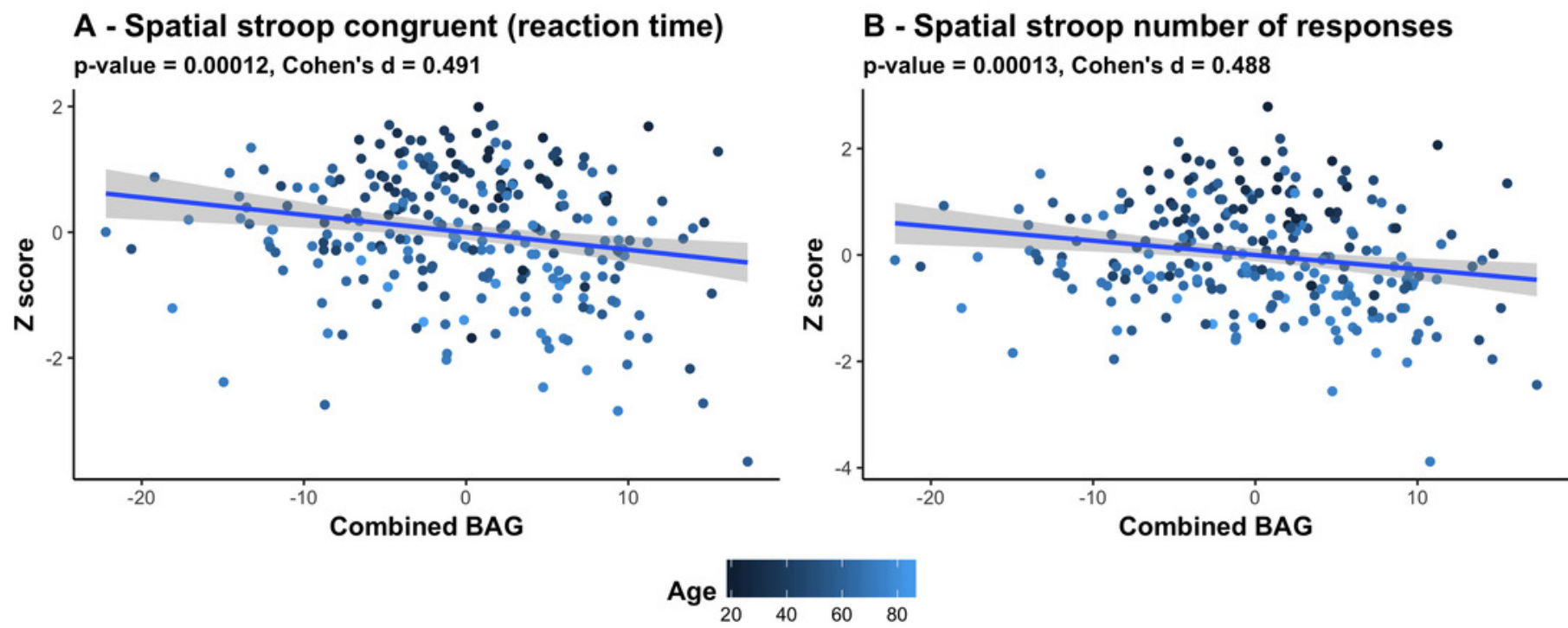


\section{Table $\mathbf{1}$ (on next page)}

Demographics and cognitive information.

* significant associations between cognitive measures with age after FDR correction, ** significant associations between cognitive measures with age after Bonferroni correction. IQR: interquartile range. MoCA: Montreal Cognitive Assessment. WASI: Wechsler Abbreviated Scale of Intelligence. CVLT: California Verbal Learning Test. STROOP: Delis-Kaplan Executive Function System (D-KEFS) color word interference test. CP: Cognitive Assessment at Bedside for IPAD (CabPAD). WM: working memory. TVA: Theory of Visual Attention. Is: longest serie. ss: sum scores. tot: total. 


\begin{tabular}{|c|c|c|c|c|c|}
\hline & Cam-CAN & $\begin{array}{l}\text { StrokeMRI } \\
\text { Mean (SD) }\end{array}$ & Range (IQR) & $\begin{array}{l}\text { Main effect Age } \\
t(p)\end{array}$ & $\begin{array}{l}\text { Main effect Sex } \\
t(p)\end{array}$ \\
\hline Total N (\% females) & $612(51.3 \%)$ & $265(63.4 \%)$ & & & \\
\hline Mean age (SD) & $54.41(18.26)$ & $56.95(14.84)$ & & & \\
\hline Age range & $18-87$ & $20-88$ & & & \\
\hline $\mathrm{MoCA}$ & - & $27.60(1.72)$ & $21-30(2)$ & $-4.57(<0.001)^{* *}$ & $-2.32(0.021)$ \\
\hline WASI words & - & $65.27(6.60)$ & $44-79(10)$ & $4.72(<0.001)^{* *}$ & $0.10(0.920)$ \\
\hline WASI matrix & - & $25.39(5.64)$ & $7-35(6)$ & $-7.60(<0.001)^{* *}$ & $-0.28(0.776)$ \\
\hline CVLT learning 1-5 & - & $48.92(11.37)$ & $17-73(15.5)$ & $-5.05(<0.001)^{* *}$ & $-5.26(<0.001)$ \\
\hline CVLT interference & - & $5.53(2.15)$ & $0-13(3)$ & $-4.33(<0.001)^{* *}$ & $-0.41(0.681)$ \\
\hline CVLT recall & - & $10.83(3.42)$ & $0-16(5)$ & $-6.50(<0.001)^{* *}$ & $5.94(<0.001)$ \\
\hline CVLT delayed recall & - & $11.39(3.44)$ & $0-16(5)$ & $-4.97(<0.001)^{* *}$ & $-5.51(<0.001)$ \\
\hline CVLT recognition hit & - & $14.70(1.50)$ & $8-16(2)$ & $-2.62(0.0093)^{*}$ & $-2.68(0.008)$ \\
\hline CVLT recognition errors & - & $3.79(3.92)$ & $0-18(4)$ & $5.22(<0.001)^{* *}$ & $4.18(<0.001)$ \\
\hline CVLT recog misses & - & $1.30(1.49)$ & $0-8(2)$ & $2.62(0.0093)^{*}$ & $2.68(0.008)$ \\
\hline CVLT recog false alarm & - & $2.46(3.48)$ & $0-18(3)$ & $4.45(<0.001)^{* *}$ & $3.59(0.0004)$ \\
\hline CVLT recog correct rejection & - & $44.20(3.92)$ & $30-48(4)$ & $-5.22(<0.001)^{* *}$ & $-4.18(<0.001)$ \\
\hline CVLT d' & - & $2.97(0.72)$ & $0.97-3.90(1.11)$ & $-5.01(<0.001)^{* *}$ & $-4.50(<0.001)$ \\
\hline STROOP 1 & - & $31.14(5.66)$ & $21-50(7)$ & $5.05(<0.001)^{* *}$ & $2.44(0.015)$ \\
\hline STROOP 2 & - & $22.12(3.49)$ & $14-35(4)$ & $2.89(0.004)^{*}$ & $2.27(0.024)$ \\
\hline STROOP 3 & - & $55.86(14.13)$ & $10-108(15)$ & $7.55(<0.001)^{* *}$ & $2.97(0.003)$ \\
\hline STROOP 4 & - & $61.74(14.85)$ & $33-117(19)$ & $7.51(<0.001)^{* *}$ & $1.77(0.078)$ \\
\hline STROOP mean 1 and 2 & - & $26.54(4.16)$ & $18.5-42(5)$ & $4.47(<0.001)^{* *}$ & $2.47(0.014)$ \\
\hline $\begin{array}{l}\text { STROOP } 3 \text { minus mean } 1 \\
\text { and } 2\end{array}$ & - & $81.94(16.51)$ & $34.5-145(18.5)$ & $7.31(<0.001)^{* *}$ & $3.02(0.003)$ \\
\hline $\begin{array}{l}\text { STROOP } 4 \text { minus mean } 1 \\
\text { and } 2\end{array}$ & - & $87.64(16.73)$ & $53.5-142(24)$ & $7.52(<0.001)^{* *}$ & $1.85(0.066)$ \\
\hline $\mathrm{CP}$ - Right motor speed & - & $79.56(23.34)$ & $34-153(32)$ & $-12.25(<0.001)^{* *}$ & $-0.36(0.716)$ \\
\hline $\mathrm{CP}$ - Left motor speed & - & $81.36(17.80)$ & $39-131(26)$ & $-12.07(<0.001)^{* *}$ & $0.20(0.842)$ \\
\hline CP - FAS Phonological flow & - & $54.70(14.53)$ & $14-95(19.75)$ & $-0.61(0.541)$ & $-2.58(0.011)$ \\
\hline
\end{tabular}




\begin{tabular}{|c|c|c|c|c|c|}
\hline $\mathrm{CP}-\mathrm{FAS}$ Semantic flow & - & $51.00(10.14)$ & $27-81(13)$ & $-2.93(0.004)^{*}$ & $-3.93(<0.001)$ \\
\hline $\mathrm{CP}$ - Visual WM forward ls & - & $4.23(1.01)$ & $2-7(2)$ & $-5.31(<0.001)^{* *}$ & $0.29(0.774)$ \\
\hline $\mathrm{CP}$ - Visual WM forward ss & - & $5.45(1.87)$ & $1-10(3)$ & $-6.59(<0.001)^{* *}$ & $-0.25(0.803)$ \\
\hline $\mathrm{CP}$ - Visual WM backward & - & $3.80(1.28)$ & $0-8(1)$ & $-4.60(<0.001)^{* *}$ & $-1.85(0.065)$ \\
\hline 1s & & & & & \\
\hline $\mathrm{CP}$ - Visual WM backward & - & $4.56(2.08)$ & $0-12(3)$ & $-5.48(<0.001)^{* *}$ & $-1.02(0.309)$ \\
\hline ss & & & & & \\
\hline $\mathrm{CP}$ - Visual WM ss & - & $9.96(3.57)$ & $1-21(4)$ & $-7.04(<0.001)^{* *}$ & $-0.95(0.342)$ \\
\hline $\begin{array}{l}\mathrm{CP}-\text { Spatial stroop } \\
\text { congruent }(\mathrm{ms})\end{array}$ & - & $674.42(132.77)$ & $410-1159(181)$ & $8.52(<0.001)^{* *}$ & $-1.03(0.304)$ \\
\hline $\begin{array}{l}\mathrm{CP}-\text { Spatial stroop } \\
\text { incongruent }(\mathrm{ms})\end{array}$ & - & $929.52(198.01)$ & $462-1827(269)$ & $9.41(<0.001)^{* *}$ & $-0.75(0.451)$ \\
\hline $\mathrm{CP}-$ Spatial stroop Errors & - & $2.17(2.41)$ & $0-11(3)$ & $0.73(0.463)$ & $1.59(0.113)$ \\
\hline $\begin{array}{l}\mathrm{CP}-\text { Spatial stroop numb of } \\
\text { reps }\end{array}$ & - & $119.63(16.64)$ & $55-166(22)$ & $-9.67(<0.001)^{* *}$ & $1.23(0.219)$ \\
\hline $\begin{array}{l}\mathrm{CP}-\text { Spatial stroop incong - } \\
\text { cong }(\mathrm{ms})\end{array}$ & - & $252(110)$ & $20-678(134.5)$ & $5.73(<0.001)^{* *}$ & $-0.68(0.498)$ \\
\hline $\mathrm{CP}-$ Spatspan 1s & - & $5.37(1.78)$ & $1-10(2)$ & $-9.12(<0.001)^{* *}$ & $-4.88(<0.001)$ \\
\hline $\mathrm{CP}$ - Spatspan tot & - & $29.87(12.43)$ & $3-55(18)$ & $-9.28(<0.001)^{* *}$ & $-4.66(<0.001)$ \\
\hline $\mathrm{CP}-$ Coding corr & - & $54.50(12.11)$ & $24-88(16)$ & $-16.69(<0.001)^{* *}$ & $-2.46(0.015)$ \\
\hline $\mathrm{CP}-$ Coding error & - & $0.67(0.99)$ & $0-5(1)$ & $-1.10(0.271)$ & $1.56(0.121)$ \\
\hline $\begin{array}{l}\text { TVA - Short-term memory } \\
\text { storage }(K)\end{array}$ & - & $3.38(0.77)$ & $1.46-5.53(1.09)$ & $-7.75(<0.001)^{* *}$ & $-1.52(0.129)$ \\
\hline TVA - Processing speed $(C)$ & - & $31.55(14.07)$ & $5.99-89.67(14.75)$ & $-4.69(<0.001)^{* *}$ & $0.41(0.6847)$ \\
\hline $\begin{array}{l}\text { TVA - Perceptual threshold } \\
\left(t_{0}\right)\end{array}$ & - & $23.01(14.05)$ & $0-79.75(17.59)$ & $5.72(<0.001)^{* *}$ & $-1.94(0.053)$ \\
\hline TVA - Error rate & - & $0.10(0.06)$ & $0.0035-0.3316(0.0983)$ & $-1.35(0.177)$ & $0.67(0.502)$ \\
\hline Cluster 1 & - & - & - & $-7.19(<0.001)^{* *}$ & $-5.16(<0.001)$ \\
\hline Cluster 2 & - & - & - & $-7.28(<0.001)^{* *}$ & $1.61(0.110)$ \\
\hline Cluster 3 & - & - & - & $-2.01(0.045)^{*}$ & $-3.99(<0.001)$ \\
\hline Cluster 4 & - & - & - & $-9.98(<0.001)^{* *}$ & $1.25(0.212)$ \\
\hline Cluster 5 & - & - & - & $-6.86(<0.001)^{* *}$ & $-2.56(0.011)$ \\
\hline Cluster 6 & - & - & - & $-15.79(<0.001)^{* *}$ & $-1.08(0.282)$ \\
\hline Cluster 7 & - & - & - & $-6.50(<0.001)^{* *}$ & $-0.77(0.440)$ \\
\hline
\end{tabular}


3 Table 1. Demographics and cognitive information. * significant associations between cognitive

4 measures with age after FDR correction, ** significant associations between cognitive measures 5 with age after Bonferroni correction. IQR: interquartile range. MoCA: Montreal Cognitive 6 Assessment. WASI: Wechsler Abbreviated Scale of Intelligence. CVLT: California Verbal 7 Learning Test. STROOP: Delis-Kaplan Executive Function System (D-KEFS) color word 8 interference test. CP: CabPad. WM: working memory. TVA: Theory of Visual Attention. 1s: 9 longest serie. ss: sum scores. tot: total. 


\section{Table 2 (on next page)}

Cognitive associations with Brain Age Gap (BAG) - statistics.

* FDR significant ** Bonferroni significant. MoCA: Montreal Cognitive Assessment. WASI: Wechsler Abbreviated Scale of Intelligence. CVLT: California Verbal Learning Test. STROOP: Delis-Kaplan Executive Function System (D-KEFS) color word interference test. CP: Cognitive Assessment at Bedside for iPAD (CabPAD). WM: working memory. TVA: Theory of Visual Attention. Is: longest serie. ss: sum scores. tot: total. 
1

\begin{tabular}{|c|c|c|c|c|c|c|}
\hline Test & Adj $R^{2}$ no-BAG & BAG & $\begin{array}{l}\text { Main effect Age } \\
t(p)\end{array}$ & $\begin{array}{l}\text { Main effect Sex } \\
t(p)\end{array}$ & $\begin{array}{l}\text { Main effect BAG } \\
t(p)\end{array}$ & $\operatorname{Adj} R^{2}$ \\
\hline \multirow[t]{3}{*}{$\mathrm{MoCA}$} & 0.0907 & $\mathrm{~T} 1$ & $-4.5596(<0.001)$ & $-2.3145(0.021)$ & $-0.124(0.901)$ & 0.0878 \\
\hline & & DTI & $-4.5599(<0.001)$ & $-2.3155(0.021)$ & $1.5914(0.113)$ & 0.0966 \\
\hline & & Combined & $-4.5653(<0.001)$ & $-2.3176(0.021)$ & $-0.4626(0.644)$ & 0.0885 \\
\hline \multirow[t]{3}{*}{ WASI words } & 0.0731 & $\mathrm{~T} 1$ & $4.7118(<0.001)$ & $0.1020(0.919)$ & $-0.2169(0.828)$ & 0.0704 \\
\hline & & DTI & $4.7056(<0.001)$ & $0.1121(0.911)$ & $-0.8126(0.417)$ & 0.0727 \\
\hline & & Combined & $4.7091(<0.001)$ & $0.1041(0.917)$ & $-0.4827(0.630)$ & 0.0711 \\
\hline \multirow[t]{3}{*}{ WASI matrix } & 0.1791 & $\mathrm{~T} 1$ & $-7.6061(<0.001)$ & $-0.2785(0.781)$ & $-0.9158(0.361)$ & 0.1793 \\
\hline & & DTI & $-7.6610(<0.001)$ & $-0.2624(0.793)$ & $-1.6546(0.099)$ & 0.1854 \\
\hline & & Combined & $-7.6128(<0.001)$ & $-0.2726(0.785)$ & $-1.1102(0.268)$ & 0.1806 \\
\hline \multirow[t]{3}{*}{ CVLT learning 1-5 } & 0.1810 & $\mathrm{~T} 1$ & $-5.0373(<0.001)$ & $-5.2514(<0.001)$ & $-0.2505(0.802)$ & 0.1750 \\
\hline & & DTI & $-5.0418(<0.001)$ & $-5.2533(<0.001)$ & $-0.3608(0.719)$ & 0.1753 \\
\hline & & Combined & $-5.0387(<0.001)$ & $-5.2522(<0.001)$ & $-0.2492(0.803)$ & 0.1750 \\
\hline \multirow[t]{3}{*}{ CVLT interference } & 0.0664 & $\mathrm{~T} 1$ & $-4.3256(<0.001)$ & $-0.4062(0.685)$ & $-0.9588(0.339)$ & 0.0626 \\
\hline & & DTI & $-4.3218(<0.001)$ & $-0.4104(0.682)$ & $-0.2391(0.811)$ & 0.0594 \\
\hline & & Combined & $-4.3202(<0.001)$ & $-0.4101(0.682)$ & $-0.1875(0.851)$ & 0.0594 \\
\hline \multirow[t]{3}{*}{ CVLT recall } & 0.2438 & $\mathrm{~T} 1$ & $-6.4897(<0.001)$ & $-5.9257(<0.001)$ & $-0.4868(0.627)$ & 0.2397 \\
\hline & & DTI & $-6.4885(<0.001)$ & $-5.9257(<0.001)$ & $-0.1245(0.901)$ & 0.2391 \\
\hline & & Combined & $-6.5080(<0.001)$ & $-5.9373(<0.001)$ & $-1.1114(0.268)$ & 0.2427 \\
\hline \multirow[t]{3}{*}{ CVLT delayed recall } & 0.1850 & $\mathrm{~T} 1$ & $-4.9636(<0.001)$ & $-5.4973(<0.001)$ & $0.1421(0.887)$ & 0.1808 \\
\hline & & DTI & $-4.9611(<0.001)$ & $-5.4969(<0.001)$ & $0.224(0.823)$ & 0.1809 \\
\hline & & Combined & $-4.9655(<0.001)$ & $-5.4954(<0.001)$ & $-0.3038(0.762)$ & 0.1810 \\
\hline \multirow[t]{3}{*}{ CVLT recognition hits } & 0.0494 & $\mathrm{~T} 1$ & $-2.6125(0.010)$ & $-2.6822(0.008)$ & $-0.8586(0.391)$ & 0.0486 \\
\hline & & DTI & $-2.6144(0.010)$ & $-2.6786(0.008)$ & $0.0946(0.925)$ & 0.0459 \\
\hline & & Combined & $-2.6212(0.009)$ & $-2.6854(0.008)$ & $-1.0724(0.285)$ & 0.0501 \\
\hline \multirow[t]{3}{*}{ CVLT recognition errors } & 0.1526 & $\mathrm{~T} 1$ & $5.2227(<0.001)$ & $4.1850(<0.001)$ & $-0.8471(0.398)$ & 0.1528 \\
\hline & & DTI & $5.2115(<0.001)$ & $4.1755(<0.001)$ & $-0.5651(0.573)$ & 0.1514 \\
\hline & & Combined & $5.2139(<0.001)$ & $4.1740(<0.001)$ & $-0.2537(0.800)$ & 0.1506 \\
\hline \multirow[t]{3}{*}{ CVLT recog misses } & 0.0494 & $\mathrm{~T} 1$ & $2.6125(0.010)$ & $2.6822(0.008)$ & $0.8586(0.391)$ & 0.0486 \\
\hline & & DTI & $2.6144(0.010)$ & $2.6786(0.008)$ & $-0.0946(0.925)$ & 0.0459 \\
\hline & & Combined & $2.6212(0.009)$ & $2.6854(0.008)$ & $1.0724(0.285)$ & 0.0501 \\
\hline \multirow[t]{3}{*}{ CVLT recog false alarm } & 0.1150 & $\mathrm{~T} 1$ & $4.4519(<0.001)$ & $3.5827(<0.001)$ & $-0.776(0.439)$ & 0.1146 \\
\hline & & DTI & $4.4378(<0.001)$ & $3.5803(<0.001)$ & $-0.5207(0.603)$ & 0.1134 \\
\hline & & Combined & $4.4418(<0.001)$ & $3.5788(<0.001)$ & $-0.3488(0.728)$ & 0.1129 \\
\hline \multirow[t]{3}{*}{ CVLT recog correct rejection } & 0.1526 & $\mathrm{~T} 1$ & $-5.2227(<0.001)$ & $-4.1850(<0.001)$ & $0.8471(0.398)$ & 0.1528 \\
\hline & & DTI & $-5.2115(<0.001)$ & $-4.1755(<0.001)$ & $0.5651(0.573)$ & 0.1514 \\
\hline & & Combined & $-5.2139(<0.001)$ & $-4.1740(<0.001)$ & $0.2537(0.800)$ & 0.1506 \\
\hline \multirow[t]{2}{*}{ CVLT d' } & 0.1566 & $\mathrm{~T} 1$ & $-5.0074(<0.001)$ & $-4.4914(<0.001)$ & $0.3628(0.717)$ & 0.1536 \\
\hline & & DTI & $-5.0021(<0.001)$ & $-4.4969(<0.001)$ & $0.8538(0.394)$ & 0.1556 \\
\hline
\end{tabular}




\begin{tabular}{|c|c|c|c|c|c|c|}
\hline \multirow[t]{3}{*}{ STROOP 1} & \multirow[t]{3}{*}{0.1118} & $\mathrm{~T} 1$ & $5.1466(<0.001)$ & $2.4999(0.013)$ & $2.6939(0.008)$ & 0.1299 \\
\hline & & DTI & $5.0968(<0.001)$ & $2.4769(0.014)$ & $1.6664(0.097)$ & 0.1147 \\
\hline & & Combined & $5.2111(<0.001)$ & $2.5317(0.012)$ & $3.3767(<0.001)^{*}$ & 0.1434 \\
\hline \multirow[t]{3}{*}{ STROOP 2} & \multirow[t]{3}{*}{0.0477} & $\mathrm{~T} 1$ & $2.8868(0.004)$ & $2.2619(0.025)$ & $0.1557(0.876)$ & 0.0433 \\
\hline & & DTI & $2.8768(0.004)$ & $2.2489(0.025)$ & $-0.4639(0.643)$ & 0.0440 \\
\hline & & Combined & $2.8949(0.004)$ & $2.2713(0.024)$ & $0.4976(0.619)$ & 0.0442 \\
\hline \multirow[t]{3}{*}{ STROOP 3} & \multirow[t]{3}{*}{0.2104} & $\mathrm{~T} 1$ & $7.5930(<0.001)$ & $2.9898(0.003)$ & $1.5092(0.133)$ & 0.2109 \\
\hline & & DTI & $7.6511(<0.001)$ & $3.0224(0.003)$ & $2.231(0.027)$ & 0.2190 \\
\hline & & Combined & $7.6793(<0.001)$ & $3.0233(0.003)$ & $2.5768(0.011)$ & 0.2240 \\
\hline \multirow[t]{3}{*}{ STROOP 4} & \multirow[t]{3}{*}{0.1887} & $\mathrm{~T} 1$ & $7.5403(<0.001)$ & $1.7884(0.075)$ & $1.2397(0.216)$ & 0.1906 \\
\hline & & DTI & $7.5847(<0.001)$ & $1.8121(0.071)$ & $1.7368(0.084)$ & 0.1953 \\
\hline & & Combined & $7.6387(<0.001)$ & $1.8247(0.069)$ & $2.3662(0.019)$ & 0.2033 \\
\hline \multirow[t]{3}{*}{ STROOP mean 1 and 2} & \multirow[t]{3}{*}{0.0949} & $\mathrm{~T} 1$ & $4.5089(<0.001)$ & $2.5033(0.013)$ & $1.5875(0.114)$ & 0.0978 \\
\hline & & DTI & $4.4750(<0.001)$ & $2.4760(0.014)$ & $0.3927(0.695)$ & 0.0894 \\
\hline & & Combined & $4.5432(<0.001)$ & $2.5399(0.012)$ & $2.0254(0.044)$ & 0.1034 \\
\hline \multirow[t]{3}{*}{ STROOP 3 minus mean 1 and 2} & \multirow[t]{3}{*}{0.2051} & $\mathrm{~T} 1$ & $7.3383(<0.001)$ & $3.0427(0.003)$ & $1.1397(0.256)$ & 0.2021 \\
\hline & & DTI & $7.3613(<0.001)$ & $3.0703(0.002)$ & $1.3546(0.177)$ & 0.2038 \\
\hline & & Combined & $7.4197(<0.001)$ & $3.1063(0.002)$ & $2.1881(0.030)$ & 0.2130 \\
\hline \multirow[t]{3}{*}{ STROOP 4 minus mean 1 and 2} & \multirow[t]{3}{*}{0.1936} & $\mathrm{~T} 1$ & $7.5360(<0.001)$ & $1.8671(0.063)$ & $0.8763(0.382)$ & 0.1919 \\
\hline & & DTI & $7.5297(<0.001)$ & $1.8697(0.063)$ & $0.6331(0.527)$ & 0.1907 \\
\hline & & Combined & $7.6081(<0.001)$ & $1.9215(0.056)$ & $1.7531(0.081)$ & 0.1993 \\
\hline \multirow[t]{3}{*}{$\mathrm{CP}$ - Right motor speed } & \multirow[t]{3}{*}{0.3695} & $\mathrm{~T} 1$ & $-12.2893(<0.001)$ & $-0.3592(0.720)$ & $-1.5504(0.122)$ & 0.3676 \\
\hline & & DTI & $-12.2318(<0.001)$ & $-0.3612(0.718)$ & $-0.3435(0.732)$ & 0.3620 \\
\hline & & Combined & $-12.3125(<0.001)$ & $-0.3587(0.720)$ & $-1.8139(0.071)$ & 0.3697 \\
\hline \multirow[t]{3}{*}{$\mathrm{CP}$ - Left motor speed } & \multirow[t]{3}{*}{0.3630} & $\mathrm{~T} 1$ & $-12.1437(<0.001)$ & $0.2100(0.834)$ & $-1.9945(0.047)$ & 0.3634 \\
\hline & & DTI & $-12.0669(<0.001)$ & $0.2081(0.835)$ & $-0.8704(0.385)$ & 0.3555 \\
\hline & & Combined & $-12.2516(<0.001)$ & $0.2149(0.830)$ & $-2.9047(0.004)$ & 0.3740 \\
\hline \multirow[t]{3}{*}{$\mathrm{CP}$ - FAS Semantic flow } & \multirow[t]{3}{*}{0.0840} & $\mathrm{~T} 1$ & $-2.9562(0.003)$ & $-3.9454(<0.001)$ & $-2.0826(0.038)$ & 0.0960 \\
\hline & & DTI & $-2.9607(0.003)$ & $-3.9388(<0.001)$ & $-2.0997(0.037)$ & 0.0963 \\
\hline & & Combined & $-2.9513(0.004)$ & $-3.9389(<0.001)$ & $-1.8308(0.068)$ & 0.0926 \\
\hline \multirow[t]{3}{*}{$\mathrm{CP}$ - Visual WM forward ls } & \multirow[t]{3}{*}{0.0936} & $\mathrm{~T} 1$ & $-5.3071(<0.001)$ & $0.2850(0.776)$ & $-0.5838(0.560)$ & 0.0906 \\
\hline & & DTI & $-5.3392(<0.001)$ & $0.2963(0.767)$ & $-1.7204(0.087)$ & 0.0999 \\
\hline & & Combined & $-5.3059(<0.001)$ & $0.2853(0.776)$ & $-0.3127(0.755)$ & 0.0897 \\
\hline \multirow[t]{3}{*}{$\mathrm{CP}$ - Visual WM forward ss } & \multirow[t]{3}{*}{0.1416} & $\mathrm{~T} 1$ & $-6.5795(<0.001)$ & $-0.2502(0.803)$ & $-0.2158(0.829)$ & 0.1375 \\
\hline & & DTI & $-6.6000(<0.001)$ & $-0.2448(0.807)$ & $-1.1695(0.243)$ & 0.1420 \\
\hline & & Combined & $-6.5786(<0.001)$ & $-0.2496(0.803)$ & $-0.02(0.984)$ & 0.1373 \\
\hline \multirow[t]{3}{*}{$\mathrm{CP}$ - Visual WM backward ls } & \multirow[t]{3}{*}{0.0852} & $\mathrm{~T} 1$ & $-4.5941(<0.001)$ & $-1.8511(0.065)$ & $-0.1047(0.917)$ & 0.0820 \\
\hline & & DTI & $-4.6170(<0.001)$ & $-1.8545(0.065)$ & $-1.3334(0.184)$ & 0.0884 \\
\hline & & Combined & $-4.6051(<0.001)$ & $-1.8550(0.065)$ & $-0.8013(0.424)$ & 0.0843 \\
\hline CP - Visual WM backward ss & 0.1022 & $\mathrm{~T} 1$ & $-5.4741(<0.001)$ & $-1.0181(0.310)$ & $-0.2721(0.786)$ & 0.1015 \\
\hline
\end{tabular}




\begin{tabular}{|c|c|c|c|c|c|c|}
\hline \multirow[t]{3}{*}{$\mathrm{CP}-$ Visual WM ss } & 0.1607 & $\mathrm{~T} 1$ & $-7.0322(<0.001)$ & $-0.9515(0.342)$ & $-0.3013(0.763)$ & 0.1591 \\
\hline & & DTI & $-7.0622(<0.001)$ & $-0.9511(0.342)$ & $-1.3634(0.174)$ & 0.1649 \\
\hline & & Combined & $-7.0399(<0.001)$ & $-0.9528(0.342)$ & $-0.6665(0.506)$ & 0.1603 \\
\hline \multirow[t]{3}{*}{$\mathrm{CP}-$ Spatial stroop congruent } & 0.2288 & $\mathrm{~T} 1$ & $8.6156(<0.001)$ & $-1.0080(0.314)$ & $2.1921(0.029)$ & 0.2288 \\
\hline & & DTI & $8.6687(<0.001)$ & $-1.0021(0.317)$ & $2.6995(0.007)$ & 0.2362 \\
\hline & & Combined & $8.8278(<0.001)$ & $-0.9828(0.327)$ & $3.9007(<0.001)^{* *}$ & 0.2588 \\
\hline \multirow[t]{3}{*}{$\mathrm{CP}$ - Spatial stroop incongruent } & 0.2548 & $\mathrm{~T} 1$ & $9.5489(<0.001)$ & $-0.7429(0.458)$ & $2.6569(0.008)$ & 0.2700 \\
\hline & & DTI & $9.5931(<0.001)$ & $-0.7587(0.449)$ & $2.8817(0.004)$ & 0.2735 \\
\hline & & Combined & $9.7197(<0.001)$ & $-0.7378(0.461)$ & $3.8071(<0.001)^{* *}$ & 0.2903 \\
\hline \multirow[t]{3}{*}{$\mathrm{CP}$ - Spatial stroop numb of reps } & 0.2731 & $\mathrm{~T} 1$ & $-9.7755(<0.001)$ & $1.2211(0.223)$ & $-2.2212(0.027)$ & 0.2753 \\
\hline & & DTI & $-9.8507(<0.001)$ & $1.2328(0.219)$ & $-2.9614(0.003)$ & 0.2859 \\
\hline & & Combined & $-9.9891(<0.001)$ & $1.2198(0.224)$ & $-3.8816(<0.001)^{* *}$ & 0.3027 \\
\hline \multicolumn{7}{|l|}{$\mathrm{CP}$ - Spatial stroop incong - } \\
\hline \multirow[t]{3}{*}{ cong } & 0.1012 & $\mathrm{~T} 1$ & $5.7663(<0.001)$ & $-0.6595(0.510)$ & $1.5611(0.120)$ & 0.1134 \\
\hline & & DTI & $5.7466(<0.001)$ & $-0.6678(0.505)$ & $0.9705(0.333)$ & 0.1081 \\
\hline & & Combined & $5.7568(<0.001)$ & $-0.6584(0.511)$ & $1.2056(0.229)$ & 0.1099 \\
\hline \multirow[t]{3}{*}{$\mathrm{CP}$ - Spatspan 1s } & 0.3055 & $\mathrm{~T} 1$ & $-9.1038(<0.001)$ & $-4.8656(<0.001)$ & $-0.032(0.975)$ & 0.3009 \\
\hline & & DTI & $-9.1746(<0.001)$ & $-4.9104(<0.001)$ & $-1.5749(0.117)$ & 0.3077 \\
\hline & & Combined & $-9.1043(<0.001)$ & $-4.8663(<0.001)$ & $-0.075(0.940)$ & 0.3009 \\
\hline \multirow[t]{3}{*}{$\mathrm{CP}$ - Spatspan total } & 0.3057 & $\mathrm{~T} 1$ & $-9.2664(<0.001)$ & $-4.6439(<0.001)$ & $0.1074(0.915)$ & 0.3024 \\
\hline & & DTI & $-9.3260(<0.001)$ & $-4.6815(<0.001)$ & $-1.3773(0.170)$ & 0.3076 \\
\hline & & Combined & $-9.2686(<0.001)$ & $-4.6461(<0.001)$ & $-0.0612(0.951)$ & 0.3024 \\
\hline \multirow[t]{3}{*}{$\mathrm{CP}$ - Coding corr } & 0.5387 & $\mathrm{~T} 1$ & $-16.7647(<0.001)$ & $-2.5004(0.013)$ & $-1.6149(0.108)$ & 0.5352 \\
\hline & & DTI & $-17.0893(<0.001)$ & $-2.5467(0.012)$ & $-3.3998(<0.001)^{*}$ & 0.5510 \\
\hline & & Combined & $-17.0071(<0.001)$ & $-2.5604(0.011)$ & $-3.0056(0.003)$ & 0.5467 \\
\hline \multicolumn{7}{|l|}{ TVA - Short-term memory } \\
\hline \multirow[t]{3}{*}{ storage $(K)$} & 0.2013 & $\mathrm{~T} 1$ & $-7.7691(<0.001)$ & $-1.5196(0.130)$ & $-1.1179(0.265)$ & 0.1981 \\
\hline & & DTI & $-7.8117(<0.001)$ & $-1.5383(0.125)$ & $-2.0302(0.043)$ & 0.2070 \\
\hline & & Combined & $-7.7525(<0.001)$ & $-1.5195(0.130)$ & $-0.9537(0.341)$ & 0.1970 \\
\hline \multirow[t]{3}{*}{ TVA - Perceptual threshold $\left(t_{0}\right)$} & 0.0764 & $\mathrm{~T} 1$ & $5.7303(<0.001)$ & $-1.9470(0.053)$ & $0.9617(0.337)$ & 0.1141 \\
\hline & & DTI & $5.7333(<0.001)$ & $-1.9444(0.053)$ & $1.1066(0.270)$ & 0.1152 \\
\hline & & Combined & $5.7523(<0.001)$ & $-1.9587(0.051)$ & $1.8346(0.068)$ & 0.1226 \\
\hline \multirow[t]{3}{*}{ TVA - Processing speed $(C)$} & 0.1304 & $\mathrm{~T} 1$ & $-4.6692(<0.001)$ & $0.3969(0.692)$ & $0.8093(0.419)$ & 0.0723 \\
\hline & & DTI & $-4.6800(<0.001)$ & $0.4053(0.686)$ & $0.1402(0.889)$ & 0.0699 \\
\hline & & Combined & $-4.6827(<0.001)$ & $0.3944(0.694)$ & $0.8916(0.374)$ & 0.0728 \\
\hline \multirow[t]{3}{*}{ Cluster 1} & 0.2470 & $\mathrm{~T} 1$ & $-7.1741(<0.001)$ & $-5.1567(<0.001)$ & $-0.1927(0.847)$ & 0.2440 \\
\hline & & DTI & $-7.1623(<0.001)$ & $-5.1410(<0.001)$ & $0.3683(0.713)$ & 0.2443 \\
\hline & & Combined & $-7.1805(<0.001)$ & $-5.1641(<0.001)$ & $-0.3879(0.699)$ & 0.2443 \\
\hline Cluster 2 & 0.1720 & $\mathrm{~T} 1$ & $-7.2680(<0.001)$ & $1.6030(0.110)$ & $-0.1013(0.919)$ & 0.1687 \\
\hline
\end{tabular}




\begin{tabular}{|c|c|c|c|c|c|c|}
\hline & & DTI & $-7.2785(<0.001)$ & $1.6062(0.110)$ & $-0.6549(0.513)$ & 0.1701 \\
\hline & & Combined & $-7.2740(<0.001)$ & $1.6104(0.109)$ & $-0.6382(0.524)$ & 0.1700 \\
\hline \multirow[t]{3}{*}{ Cluster 3} & 0.0698 & $\mathrm{~T} 1$ & $-2.0177(0.045)$ & $-3.9824(<0.001)$ & $-0.8103(0.419)$ & 0.0686 \\
\hline & & DTI & $-2.0337(0.043)$ & $-3.9969(<0.001)$ & $-1.84(0.067)$ & 0.0783 \\
\hline & & Combined & $-2.0185(0.045)$ & $-3.9877(<0.001)$ & $-0.9765(0.330)$ & 0.0697 \\
\hline \multirow[t]{3}{*}{ Cluster 4} & 0.2783 & $\mathrm{~T} 1$ & $-10.1319(<0.001)$ & $1.2314(0.219)$ & $-2.5436(0.012)$ & 0.2937 \\
\hline & & DTI & $-10.1479(<0.001)$ & $1.2377(0.217)$ & $-2.5207(0.012)$ & 0.2933 \\
\hline & & Combined & $-10.3013(<0.001)$ & $1.2196(0.224)$ & $-3.6163(<0.001)^{*}$ & 0.3113 \\
\hline \multirow[t]{3}{*}{ Cluster 5} & 0.1772 & $\mathrm{~T} 1$ & $-6.8872(<0.001)$ & $-2.5902(0.010)$ & $-1.1084(0.269)$ & 0.1779 \\
\hline & & DTI & $-6.8667(<0.001)$ & $-2.5805(0.010)$ & $-0.5825(0.561)$ & 0.1750 \\
\hline & & Combined & $-6.9577(<0.001)$ & $-2.6481(0.009)$ & $-1.9103(0.057)$ & 0.1858 \\
\hline \multirow[t]{3}{*}{ Cluster 6} & 0.5092 & $\mathrm{~T} 1$ & $-15.9345(<0.001)$ & $-1.1148(0.266)$ & $-1.8971(0.059)$ & 0.5145 \\
\hline & & DTI & $-15.9719(<0.001)$ & $-1.1080(0.269)$ & $-2.0875(0.038)$ & 0.5160 \\
\hline & & Combined & $-16.0156(<0.001)$ & $-1.1196(0.264)$ & $-2.459(0.015)$ & 0.5193 \\
\hline \multirow[t]{3}{*}{ Cluster 7} & 0.1399 & $\mathrm{~T} 1$ & $-6.4852(<0.001)$ & $-0.7736(0.440)$ & $-0.3433(0.732)$ & 0.1369 \\
\hline & & DTI & $-6.5210(<0.001)$ & $-0.7689(0.443)$ & $-1.6007(0.111)$ & 0.1452 \\
\hline & & Combined & $-6.4926(<0.001)$ & $-0.7759(0.439)$ & $-0.63(0.529)$ & 0.1379 \\
\hline
\end{tabular}

2 Table 2. Cognitive associations with BAG - statistics. * FDR significant ** Bonferroni

3 significant. MoCA: Montreal Cognitive Assessment. WASI: Wechsler Abbreviated Scale of

4 Intelligence. CVLT: California Verbal Learning Test. STROOP: Delis-Kaplan Executive

5 Function System (D-KEFS) color word interference test. CP: Cognitive Assessment at Bedside

6 for iPAD (CabPAD). WM: working memory. TVA: Theory of Visual Attention. 1s: longest serie.

7 ss: sum scores. tot: total. 